\title{
VOID FRACTION DISTURBANCES IN A UNIFORM BUBBLY FLUID
}

\author{
A. Biesheuvel and W. C. M. Gorissen \\ Department of Mechanical Engineering, University of Twente, P.O. Box 217, 7500 AE Enschede, \\ The Netherlands
}

(Received 27 July 1988; in revised form 2 August 1989)

\begin{abstract}
The paper is concerned with the flow of dispersions of gas bubbles in liquid, with bubble sizes such that the inertia forces on the bubbles are of importance to the dynamics. One-dimensional conservation equations are derived, which govern the flow when the deviations from the uniform state are small. These are used to describe the features of the propagation of void fraction disturbances, and to investigate the stability of uniform bubbly flows. The results are compared with what has been observed in experiments.
\end{abstract}

Key Words: bubbly flows, void fraction waves, stability

\section{INTRODUCTION}

During the past decade an experimental research programme on the propagation of void fraction waves in bubbly fluids has been carried out at the Centre d'Études Nucléaires de Grenoble by Bouré and co-workers (Mercadier 1981; Micaelli 1982; Matuszkiewicz et al. 1987). Void fraction disturbances caused by natural or imposed fluctuations of the rate of air supply were detected by means of impedance probes mounted in the walls of vertical tubes. The wave properties were deduced by correlation techniques. The observations of Mercadier (1981) of waves in bubbly fluids with mean void fractions up to $28 \%$ may be summarized as follows:

- The detected wave frequencies $v$ do not exceed a few $\mathrm{Hz}$ : for a void fraction of $8 \%, v<2 \mathrm{~Hz}$ in stagnant fluid and $v<10 \mathrm{~Hz}$ when the superficial liquid velocity is $1.0 \mathrm{~m} / \mathrm{s}$.

- The phase velocity of the waves does not significantly depend on the frequency.

- The phase velocity of the waves is less than the mean bubble velocity and larger than the mean fluid velocity.

- The attenuation of the waves decreases with increasing value of the mean void fraction.

Micaelli (1982) made measurements in highly turbulent flows, superficial liquid velocities in the range of $1-8 \mathrm{~m} / \mathrm{s}$, with mean void fractions up to $20 \%$. He concluded that the attenuation rate is proportional to the square of the wave frequency.

Matuszkiewicz et al. (1987) report similar observations for bubbly fluids with void fractions up to $35 \%$ and a fixed superficial liquid velocity of $0.18 \mathrm{~m} / \mathrm{s}$. In addition it was found that in the range of void fractions of $35-45 \%$ a smooth transition seems to occur: void fraction disturbances are either slightly damped or amplified, the experimental accuracy was not sufficient to draw conclusions. Void fraction waves were definitely unstable for void fractions $>45 \%$ and slugs could be observed in the upper part of the test section. The dominant frequency of the unstable waves showed a slight increase with the void fraction: 1.0 and $1.2 \mathrm{~Hz}$ for void fractions of 47 and $50 \%$, respectively. The authors remark that the actual appearance of gas slugs results from a weak instability of the bubbly flow and that it entails a transition length. They finally conclude that their work has substantiated the existence of a relationship between the bubbly-slug flow transition and a void fraction wave instability in the bubbly flow.

The purpose of our paper is to explain the observations of linear void fraction wave propagation by the group at the C.E.N.G. and to investigate whether a uniform bubbly flow is unstable to void fraction disturbances above some critical value of the void fraction. We shall make the simplifying 
assumptions that the bubbly dispersion is uniform in the unperturbed state, and that it consists of equally sized spherical bubbles with a diameter such that irrotational flow theory can be used to describe their motion. Only the propagation of one-dimensional planar disturbances will be considered, and wall effects will not be taken into account. The basic equations for the analysis are the conservation equations for mean number density and mean momentum of the gas bubbles. Unfortunately, there exists no agreement on the way in which transient and dynamic pressure forces exerted by the fluid, are to be incorporated in the bubble momentum equation. Therefore, a substantial part of the paper is devoted to a formal derivation of this equation. The method used is that of averaging over an ensemble of realizations of the flow, similar to that of the kinetic theory of dense gases (Hirschfelder et al. 1954; Rice \& Gray 1965). Throughout the analysis it will be assumed that the fluid flow around the bubbles is laminar; but arguments will be given that make clear that the resulting equations may also be applied to turbulent flows.

In section 2 an approximate expression for the force on a reference bubble in one realization of the flow is derived. A few relevant concepts of kinetic theory are mentioned in section 3; the averaging process leading to the required conservation equations is then performed in section 4 . The result is a momentum equation in which various flow parameters are defined in terms of the probability distribution function of the positions and velocities of the bubbles. These parameters are subsequently modelled by phenomenological reasoning in section 5 . The propagation of linear void fraction waves is the subject of section 6 and the linear stability of a uniform bubbly flow is considered in section 7 .

Two months after the first version of our paper was submitted, an article by Professor G. K. Batchelor was published (Batchelor 1988) that presents a theory of the instability of uniform fluidized beds. Our work conforms in many respects to that of Professor Batchelor, and when revising our paper it turned out that it could be improved by making use of parts of his analysis. A reference to Batchelor (1988) will be given wherever this has been done.

\section{AN EQUATION OF MOTION FOR A BUBBLE IN ONE REALIZATION OF THE FLOW}

\section{A single bubble}

Experiments have shown that a single bubble in pure water will rise rectilinearly, while maintaining an approximately spherical shape, if the Reynolds number $(\mathrm{Re})$ of the motion, based on the bubble diameter, is about 100 . A method to calculate an expression for the rise velocity is explained in Moore (1963) and Batchelor (1967; section 5.14): The flow field is approximately irrotational with vorticity being confined to a relatively thin boundary layer and wake with dimensionless thickness and diameter of $O\left(\mathrm{Re}^{-1 / 2}\right)$ and $O\left(\mathrm{Re}^{-1 / 4}\right)$, respectively. At the free surface only continuity of the tangential stress is required, and as a consequence the dimensionless velocity perturbations in the boundary layer and the wake are only of $O\left(\operatorname{Re}^{-1 / 2}\right)$. This allows one to determine a first-order approximation to the drag coefficient of the bubble from a calculation of the rate of dissipation in the fluid, based on the assumption that the flow field is wholly irrotational. Equating this to the rate of work done by the drag force yields

$$
C_{\mathrm{d}}=\frac{48}{\mathrm{Re}}+O\left(\mathrm{Re}^{-3 / 2}\right)
$$

The frictional force $F_{\mathrm{f}_{x}}$ and the velocity of rise $v_{\infty}$ are then given by

$$
F_{\mathrm{f}_{\mathrm{x}}}=-12 \pi a \mu_{\mathrm{L}} v_{\infty}
$$

and

$$
v_{\infty}=\frac{\left(\rho_{\mathrm{L}}-\rho_{\mathrm{G}}\right) g a^{2}}{9 \mu_{\mathrm{L}}},
$$


where $a$ is the bubble radius, $\mu_{\mathrm{L}}$ is the liquid viscosity and $g$ is the gravitational accelerator; $\rho_{\mathrm{L}}$ and $\rho_{\mathrm{G}}$ denote the liquid density and gas density, respectively. As a typical example: a bubble with radius $a=0.4 \mathrm{~mm}$ would rise in water (at room temperature) with a velocity $v_{\infty}=17.4 \mathrm{~cm} / \mathrm{s}$ with corresponding Reynolds number, $\mathrm{Re}=140$.

An expression for the force exerted by the fluid when a bubble is in unsteady motion can be found by the following argument (Lighthill 1986; section 8.3): The irrotational flow can at each instant be imagined to have been set-up from rest by a large impulsive force $F$ acting on the bubble over a very small time $\tau$. The total impulse imparted to the bubble and the fluid, the Kelvin impulse I, is given by the time integral of the force

$$
\mathbf{I}=\int_{0}^{\tau} \mathbf{F} \mathrm{d} t,
$$

which is equal to

$$
\mathbf{I}=\frac{4}{3} \pi a^{3} \rho_{\mathrm{G}} \mathbf{v}+\mathbf{I}_{\mathrm{L}},
$$

where $\mathbf{v}$ is the bubble velocity at the moment of consideration and the fluid impulse $I_{L}$ is defined by

$$
\mathbf{I}_{\mathrm{L}}=-\rho_{\mathrm{L}} \int \phi \mathbf{n} \mathrm{d} A .
$$

Here $\phi$ is the velocity potential of the irrotational flow, and $\mathrm{d} A$ is a surface element coinciding with the surface of the bubble, with unit normal $\mathbf{n}$ directed into the fluid. Solving the potential flow problem shows that the fluid impulse may be written as

$$
\mathbf{I}_{\mathrm{L}}=m_{\infty} \mathbf{v}, \text { with } m_{\infty}=\frac{2}{3} \pi a^{3} \rho_{\mathrm{L}} .
$$

This suggests that the presence of the fluid effectively increases the mass of the bubbles by a term $m_{\infty}$, the added mass. Alternatively, the fluid impulse may be interpreted as an addition to the momentum of the bubble. The change in the Kelvin impulse in a very small time $\mathrm{d} t$ can be written as

$$
\begin{aligned}
d \mathbf{I} & =\mathrm{d}\left(\frac{4}{3} \pi a^{3} \rho_{\mathrm{G}} \mathbf{v}+\mathbf{I}_{\mathrm{L}}\right) \\
& =\int_{0}^{\tau+\mathrm{d} t} \mathbf{F} \mathrm{d} t-\int_{0}^{\tau} \mathbf{F} \mathrm{d} t=\mathbf{F} \mathrm{d} t .
\end{aligned}
$$

It follows that if the fluid were inviscid, the force exerted on the bubble would be equal to minus the rate of change of the fluid impulse.

The result can also be found from an energy argument. The velocity of the bubble increases during the very short time interval $\tau$ like $(t / \tau) \mathrm{v}$; and the kinetic energy transmitted by the force $\mathbf{F}$ is given by the time integral

$$
T=\int_{0}^{\tau}\left(\frac{t}{\tau}\right) \mathbf{v} \cdot \mathbf{F} \mathrm{d} t=\frac{1}{2} \mathbf{v} \cdot \mathbf{F} \tau=\frac{1}{2} \mathbf{v} \cdot \mathbf{I} .
$$

So, over a short interval $\mathrm{d} t$ the change in the kinetic energy will be

$$
\mathrm{d} T=\mathrm{d}\left(\frac{1}{2}\left(\frac{4}{3} \pi a^{3} \rho_{\mathrm{G}}\right) \mathbf{v} \cdot \mathbf{v}+\frac{1}{2} \mathbf{v} \cdot \mathbf{I}_{\mathrm{L}}\right) .
$$

In the absence of external forces it is then found from Lagrange's equation that

$$
\frac{\mathrm{d}}{\mathrm{d} t} \nabla_{\mathbf{v}}\left(\frac{1}{2}\left(\frac{4}{3} \pi a^{3} \rho_{\mathrm{G}}\right) \mathbf{v} \cdot \mathbf{v}\right)=-\frac{\mathrm{d}}{\mathrm{d} t} \nabla_{\mathbf{v}}\left(\frac{1}{2} \mathbf{v} \cdot \mathbf{I}_{\mathrm{L}}\right)
$$

corresponding with what was found above.

The viscosity of the fluid gives rise to a frictional force that is approximately given by [1], but with $v_{\infty}$ now replaced by the instantaneous value of the velocity. The changes in the vorticity distribution of the rotational flow regions due to the unsteady motion of the bubble will also cause 
a force, but this contribution is usually taken to be of the same order as the terms that are neglected in [1]. Thus, we finally obtain the equation of motion:

$$
\frac{\mathrm{d}}{\mathrm{d} t}\left(\frac{4}{3} \pi a^{3} \rho_{\mathrm{G}} \mathbf{v}\right)=\frac{\mathrm{d}}{\mathrm{d} t}\left(\mathbf{I}_{\mathrm{L}}\right)-12 \pi a \mu_{\mathrm{L}} \mathbf{v}+\frac{4}{3} \pi a^{3}\left(\rho_{\mathrm{G}}-\rho_{\mathrm{L}}\right) \boldsymbol{g}
$$

\section{A bubble in a dispersion}

We now assume that the methods presented above can also be used in a derivation of an equation of motion for a "reference bubble" in a bubbly dispersion. The velocity field outside the boundary layer attached to the reference sphere will not be wholly irrotational due to the presence of vorticity that is produced in the boundary layers of bubbles that have passed through the region of location of the reference sphere. Although the velocity fluctuations in these rotational parts of the flow will still be small, they may give rise to a significant contribution to the total rate of energy dissipation, because the volume of the rotational flow regions may, particularly for large void fractions, not be negligibly small in comparison with that of the irrotational part of the flow. Still, for want of something better we determine the forces on a reference bubble of a dispersion in a manner analogous to that employed in the case of a single bubble.

To this end, consider an element of the dispersion of volume $V$, containing a large number of bubbles $N, N \gg 1$. The positions $\mathbf{x}_{k}$ and velocities $\dot{\mathbf{x}}_{k}, k=1, \ldots, N$, of the bubbles will be defined with respect to a frame of reference in which there is no net volume flux, i.e. a frame moving with the mean velocity of the dispersion. If it is assumed that the dispersion is uniform this velocity is specified by the volume fluxes of gas and liquid that are supplied to the system. In this paper only one-dimensional, planar void fraction disturbances to a uniform dispersion will be considered. In such a case, if the gas and liquid supply remain unchanged the mean volume flux is constant, on account of the incompressibility of the two phases; changes in the volume flux of gas are compensated by changes in the liquid volume flux. It is this situation that is envisaged in the following derivations. When, as in the experiments, the planar disturbances are a result of changes in the supply of either of the phases the mean velocity is merely a function of time. It is not difficult to make allowance for this effect in the resulting equations of motion; the pertinent equations will be given at the end of section 5 .

In the absence of any forces, including gravitational forces, the bubbles and the fluid will move with a uniform velocity that is determined by the volume fluxes of the two phases as supplied to the system; this velocity is equal to the mean velocity of the dispersion and so with respect to the chosen reference frame the system can be considered "at rest". Then, in the actual situation, at any given instant the irrotational part of the velocity field can be imagined to have been set-up instantaneously from "rest" by the action over a very short time of large impulsive forces on the bubbles, and on the material boundary of the element. This boundary, $\delta A$ say, will, if randomly chosen, consist both of particles of fluid and of gas. The kinetic energy, transmitted to the element of the dispersion by the action of the distribution of impulsive forces, is equal to

$$
\sum_{k=1}^{N}\left(\frac{1}{2} \dot{\mathbf{x}}_{k} \cdot \mathbf{I}_{k}\right)+\int_{\delta A} \rho \phi \nabla \phi \cdot \mathbf{n d} A .
$$

Here the unit normal $\mathbf{n}$ is taken to direct outwards from the element and $\rho$ is either equal to the gas density or to the liquid density, depending on whether the surface element $\mathbf{n} \mathrm{d} A$ coincides with a material surface element that consists of gas particles or liquid particles. In analogy with the case of a single bubble the "Kelvin impulse associated with bubble $k$ " is

$$
\mathbf{I}_{k}=\frac{4}{3} \pi a^{3} \rho_{\mathrm{G}} \dot{\mathbf{x}}_{k}+\mathbf{I}_{\mathrm{L}_{k}}
$$

with a "fluid impulse associated with bubble $k$ " defined by

$$
\mathbf{I}_{\mathrm{L}_{k}}=-\rho_{\mathrm{L}} \int \phi \mathrm{d} A_{k} \text {. }
$$

This fluid impulse of bubble $k$ will be a linear function of the velocities of the spheres and will also depend on their relative positions. When we let the volume element be indefinitely large, then at any instant the contribution from the integral over the boundary $\delta A$ to [7] will become very small 
compared to that of the first term. It follows that, if the fluid is assumed to be inviscid, the equation of motion of bubble $j$ is given by Lagrange's equation (Goldstein 1980, pp. 20-21):

$$
\frac{\mathrm{d}}{\mathrm{d} t}\left(\nabla_{\mathbf{x}_{j}}(T-U)\right)-\nabla_{\mathbf{x}_{j}}(T-U)=0,
$$

where $T$ and $U$ are defined by

$$
T=\sum_{k=1}^{N} \frac{1}{2}\left(\frac{4}{3} \pi a^{3} \rho_{\mathrm{G}}\right) \dot{\mathbf{x}}_{k} \cdot \dot{\mathbf{x}}_{k}
$$

and

$$
U=-\sum_{k=1}^{N} \frac{1}{2} \dot{\mathbf{x}}_{k} \cdot \mathbf{I}_{L_{k}}
$$

If we assume, as for a single bubble, that the flow is wholly irrotational, the rate of energy dissipation through the action of viscosity can be expressed as (Batchelor 1967, section 5.14)

$$
\mathfrak{D}=\mu_{\mathrm{L}} \sum_{k=1}^{N} \int \mathrm{d} \mathbf{A}_{k} \cdot \nabla\left(\frac{\partial \phi}{\partial x_{i}}\right)^{2}, \quad i=1,2,3
$$

The frictional force on bubble $j$ will be a linear function of the bubble velocities, and we may therefore derive it in terms of Rayleigh's dissipation function $\mathfrak{F}$, defined as half the rate of energy dissipation (Goldstein 1980, p. 24):

$$
\mathbf{F}_{\mathrm{f}_{j}}=-\nabla_{\dot{\mathbf{x}}_{j}} \boldsymbol{\nabla}=\nabla_{\dot{\mathrm{x}}_{j}}\left(-\frac{1}{2} \mu_{\mathrm{L}} \sum_{k=1}^{N} \int \mathrm{d} \mathbf{A}_{k} \cdot \nabla\left(\frac{\partial \phi}{\partial x_{i}}\right)^{2}\right) .
$$

If we further include the gravitational force and the buoyancy force the (approximate) equation of motion for reference bubble $j$ finally becomes, upon evaluation of [10]:

$$
\frac{\mathrm{d}}{\mathrm{d} t} \mathbf{I}_{j}=\nabla_{\mathbf{x}_{j}}\left(\sum_{k=1}^{N} \frac{1}{2} \dot{\mathbf{x}}_{k} \cdot \mathbf{I}_{\mathbf{L}_{k}}\right)+\mathbf{F}_{\mathrm{f}_{j}}+\frac{4}{3} \pi a^{3}\left(\rho_{\mathrm{G}}-\rho_{\mathrm{L}}\right) \mathbf{g}
$$

where the impulses and the frictional force are defined by [8], [9] and [11].

\section{ELEMENTS OF KINETIC THEORY}

The method of averaging that will be employed in the next section for the derivation of the equations of motion of the bubbles is that of averaging over an ensemble of realizations of the flow. To this end a probability density function $f^{N}\left(\mathscr{C}_{N}, \dot{\mathscr{C}}_{N}, t\right)$ is introduced such that

$f^{N}\left(\mathscr{C}_{N}, \dot{\mathscr{C}}_{N}, t\right) \delta \mathscr{C}_{N}, \delta \dot{\mathscr{C}}_{N}=f^{N}\left(\mathbf{x}_{1}, \mathbf{x}_{2}, \ldots, \mathbf{x}_{N}, \dot{\mathbf{x}}_{1}, \dot{\mathbf{x}}_{2}, \ldots, \dot{\mathbf{x}}_{N}, t\right) \delta \mathbf{x}_{1}, \delta \mathbf{x}_{2}, \ldots, \delta \mathbf{x}_{N}, \delta \dot{\mathbf{x}}_{1}, \delta \dot{\mathbf{x}}_{2}, \ldots, \delta \dot{\mathbf{x}}_{N}$

is the probability of finding $N$ bubbles, where $N \gg 1$, at time $t$ in the volume elements $\delta \mathbf{x}_{1}, \delta \mathbf{x}_{2}, \ldots, \delta \mathbf{x}_{N}, \delta \dot{\mathbf{x}}_{1}, \delta \dot{\mathbf{x}}_{2}, \ldots, \delta \dot{\mathbf{x}}_{N}$ around the points $\mathbf{x}_{1}, \mathbf{x}_{2}, \ldots, \mathbf{x}_{N}, \dot{\mathbf{x}}_{1}, \dot{\mathbf{x}}_{2}, \ldots, \dot{\mathbf{x}}_{N}$ of $6 \mathrm{~N}-$ dimensional phase space. A point in this space specifies the positions $\mathbf{x}_{k}, k=1,2, \ldots, N$, and velocities $\dot{\mathbf{x}}_{k}$ of the $N$ bubbles in one realization of the flow. Since the bubbles are assumed to be identical rigid spheres this probability density function is normalized by

$$
\iint f^{N}\left(\mathscr{C}_{N}, \dot{\mathscr{C}}_{N}, t\right) \mathrm{d} \mathscr{C}_{N} \mathrm{~d} \dot{\mathscr{C}}_{N}=N !
$$


Mean flow parameters such as the mean number density $n(\mathbf{x}, t)$, the mean void fraction $\epsilon(\mathbf{x}, t)$ and the mean bubble velocity $\mathbf{v}(\mathbf{x}, t)$ are defined by

$$
\begin{gathered}
n(\mathbf{x}, t)=\frac{1}{N !} \iint \sum_{k=1}^{N} \delta\left(\mathbf{x}_{k}-\mathbf{x}\right) f^{N}\left(\mathscr{C}_{N}, \dot{\mathscr{C}}_{N}, t\right) \mathrm{d} \mathscr{C}_{N} \mathrm{~d} \mathscr{C}_{N}, \\
\epsilon(\mathbf{x}, t)=\frac{4}{3} \pi a^{3} n(\mathbf{x}, t)
\end{gathered}
$$

and

$$
n(\mathbf{x}, t) \mathbf{v}(\mathbf{x}, t)=\frac{1}{N !} \iint \sum_{k=1}^{N} \dot{\mathbf{x}}_{k} \delta\left(\mathbf{x}_{k}-\mathbf{x}\right) f^{N}\left(\mathscr{C}_{N}, \dot{\mathscr{C}}_{N}, t\right) \mathrm{d} \mathscr{C}_{N} \mathrm{~d} \dot{\mathscr{C}}_{N}
$$

definitions of other relevant flow parameters will be given in the next section.

The distribution function $f^{N}$ satisfies the Liouville equation

$$
\frac{\partial f^{N}}{\partial t}+\sum_{k=1}^{N}\left[\nabla_{\mathbf{x}_{k}} \cdot\left(\dot{\mathbf{x}}_{k} f^{N}\right)+\nabla_{\dot{\mathbf{x}}_{k}} \cdot\left(\ddot{\mathbf{x}}_{k} f^{N}\right)\right]=\mathbf{0}
$$

which merely expresses that systems constituting the ensemble are neither created nor destroyed. From the Liouville equation one can derive the so-called general equation of change, which in textbooks on the kinetic theory of dense gases is often taken as a starting point for the derivation of the conservation equations of hydrodynamics (Hirschfelder et al. 1954; Rice \& Gray 1965):

Let $\beta\left(\mathbf{x}_{1}, \mathbf{x}_{2}, \ldots, \mathbf{x}_{N}, \dot{\mathbf{x}}_{1}, \dot{\mathbf{x}}_{2}, \ldots, \dot{\mathbf{x}}_{N}\right)$ be a dynamical variable that does not explicitly depend on time. The ensemble mean value of $\beta$ is, as above, defined by

$$
\bar{\beta}=\frac{1}{N !} \iint \beta f^{N} \mathrm{~d} \mathscr{C}_{N} \mathrm{~d} \dot{\mathscr{C}}_{N}=\left\langle\beta ; f^{N}\right\rangle,
$$

where the latter notation using brackets is sometimes more convenient in what follows. Multiplication of the Liouville equation by $\beta$ and performing the averaging yields

$$
\frac{\partial}{\partial t}\left\langle\beta ; f^{N}\right\rangle=-\sum_{k=1}^{N}\left\langle\beta ; \nabla_{\mathbf{x}_{k}} \cdot\left(\dot{\mathbf{x}}_{k} f^{N}\right)+\nabla_{\dot{\mathbf{x}}_{k}} \cdot\left(\ddot{\mathbf{x}}_{k} f^{N}\right)\right\rangle .
$$

After partial integration over the phase space, under the assumption that $f^{N} \rightarrow 0$ sufficiently rapidly as $\left|\mathbf{x}_{1}, \ldots, \mathbf{x}_{N}, \dot{\mathbf{x}}_{1}, \ldots, \dot{\mathbf{x}}_{N}\right| \rightarrow \infty$, this finally becomes the general equation of change

$$
\frac{\partial}{\partial t}\left\langle\beta ; f^{N}\right\rangle=-\sum_{k=1}^{N}\left\langle\dot{\mathbf{x}}_{k} \cdot \nabla_{\mathbf{x}_{k}} \beta+\ddot{\mathbf{x}}_{k} \cdot \nabla_{\dot{\mathbf{x}}_{k}} \beta ; f^{N}\right\rangle .
$$

Physically relevant conservation equations can be be derived by introducing into this equation choices of $\beta$ for which the related ensemble averages can be interpretated as meaningful (observable) flow parameters.

\section{DERIVATION OF THE EQUATIONS OF MOTION}

Conservation of number density and void fraction

A conservation equation for the mean number density of the gas bubbles follows from the choice

$$
\beta=\sum_{j=1}^{N} \delta\left(\mathbf{x}_{j}-\mathbf{x}\right)
$$

For fixed $k$ we have in the r.h.s. of [16],

$$
\dot{\mathbf{x}}_{k} \cdot \nabla_{\mathbf{x}_{k}}\left(\sum_{j=1}^{N} \delta\left(\mathbf{x}_{j}-\mathbf{x}\right)\right)+\ddot{\mathbf{x}}_{k} \cdot \nabla_{\dot{\mathbf{x}}_{k}}\left(\sum_{j=1}^{N} \delta\left(\mathbf{x}_{j}-\mathbf{x}\right)\right)=\dot{\mathbf{x}}_{k} \cdot \nabla_{\mathbf{x}_{k}} \delta\left(\mathbf{x}_{j}-\mathbf{x}\right)=-\nabla_{\mathbf{x}} \cdot\left(\dot{\mathbf{x}}_{k} \delta\left(\mathbf{x}_{k}-\mathbf{x}\right)\right)
$$

Next, performing the averaging yields, upon use of the definitions [13] and [15],

$$
\frac{\partial n}{\partial t}=-\nabla_{\mathbf{x}} \cdot(n \mathbf{v})
$$


Multiplication of [17] by $4 / 3 \pi a^{3}$ gives the void fraction conservation equation

$$
\frac{\partial \epsilon}{\partial t}=-\nabla_{\mathbf{x}} \cdot(\epsilon \mathbf{v})
$$

Note that an equation equivalent to the familiar continuity equation of a fluid is found when [18] is multiplied by the gas density $\rho_{\mathrm{G}} ; \rho_{\mathrm{G}} \epsilon$ is the mass density of the bubbles.

\section{Conservation of bubble momentum and Kelvin impulse}

The analyis of section 2 suggests that a convenient choice for a derivation of a momentum equation for the bubbles is

$$
\beta=\sum_{j=1}^{N} \mathbf{I}_{j} \delta(\mathbf{x}-\mathbf{x})=\sum_{j-1}^{N}\left(\frac{4}{3} \pi a^{3} \rho_{\mathrm{G}} \dot{\mathbf{x}}_{j}+\mathbf{I}_{\mathrm{L}_{j}}\right) \delta\left(\mathbf{x}_{j}-\mathbf{x}\right) .
$$

With [19], rewriting of the general equation of change will yield an equation expressing the conservation of the mean Kelvin impulse, rather than a conservation equation for the mean bubble momentum. The derivation is straightforward but somewhat lengthy and is almost analogous to that given in Rice \& Gray (1965, section 6.2.B); the essential steps are given below.

After substitution of [19], the r.h.s. of [16] will include a term which for fixed $k$ can be rewritten as follows:

$$
\begin{aligned}
& \left(\dot{\mathbf{x}}_{k} \cdot \nabla_{\mathbf{x}_{k}}+\ddot{\mathbf{x}}_{k} \cdot \nabla_{\dot{\mathbf{x}}_{k}}\right)\left(\sum_{j=1}^{N} \mathbf{I}_{j} \delta\left(\mathbf{x}_{j}-\mathbf{x}\right)\right) \\
& =\dot{\mathbf{x}}_{k} \cdot\left(\mathbf{I}_{k} \nabla_{\mathbf{x}_{k}} \delta\left(\mathbf{x}_{k}-\mathbf{x}\right)+\sum_{j=1}^{N} \nabla_{\mathbf{x}_{j}}\left(\mathbf{I}_{j}\right) \delta\left(\mathbf{x}_{j}-\mathbf{x}\right)\right)+\sum_{j=1}^{N} \ddot{\mathbf{x}}_{k} \cdot \nabla_{\mathbf{x}_{k}}\left(\mathbf{I}_{j}\right) \delta\left(\mathbf{x}_{j}-\mathbf{x}\right) \\
& =-\nabla_{\mathbf{x}} \cdot\left(\mathbf{I}_{k} \dot{\mathbf{x}}_{k} \delta\left(\mathbf{x}_{k}-\mathbf{x}\right)\right)+\sum_{j=1}^{N}\left(\dot{\mathbf{x}}_{k} \cdot \nabla_{\mathbf{x}_{k}}+\ddot{\mathbf{x}}_{k} \cdot \nabla_{\dot{\mathbf{x}}_{k}}\right)\left(\mathbf{I}_{j}\right) \delta\left(\mathbf{x}_{j}-\mathbf{x}\right) .
\end{aligned}
$$

With this result the general equation of change becomes

$$
\begin{aligned}
\frac{\partial}{\partial t}\left\langle\sum_{k=1}^{N} \mathbf{I}_{k} \delta\left(\mathbf{x}_{k}-\mathbf{x}\right) ; f^{N}\right\rangle= & -\nabla_{\mathbf{x}} \cdot\left\langle\sum_{k=1}^{N} \mathbf{I}_{k} \dot{\mathbf{x}}_{k} \delta\left(\mathbf{x}_{k}-\mathbf{x}\right) ; f^{N}\right\rangle \\
& +\left\langle\sum_{j, k=1}^{N}\left(\dot{\mathbf{x}}_{k} \cdot \nabla_{\mathbf{x}_{k}}+\ddot{\mathbf{x}}_{k} \cdot \nabla_{\dot{\mathbf{x}}_{k}}\right)\left(\mathbf{I}_{j}\right) \delta\left(\mathbf{x}_{j}-\mathbf{x}\right) ; f^{N}\right\rangle .
\end{aligned}
$$

In the second term on the r.h.s. of [20] we may write

$$
\sum_{k=1}^{N}\left(\ddot{\mathbf{x}}_{k} \cdot \nabla_{\mathbf{x}_{k}}+\ddot{\mathbf{x}}_{k} \cdot \nabla_{\dot{\mathbf{x}}_{k}}\right) \mathbf{I}_{j}=\mathbf{I}_{j},
$$

i.e. the time rate of change of the Kelvin impulse associated with bubble $j$. Upon use of the equation of motion for a bubble in one realization of the flow, viz. [12], [21a] becomes

$$
=\nabla_{\mathbf{x}_{j}}\left(\sum_{k=1}^{N} \frac{1}{2} \dot{\mathbf{x}}_{k} \cdot \mathbf{I}_{\mathrm{L}_{k}}\right)+\mathbf{F}_{\mathrm{F}_{j}}+\frac{4}{3} \pi a^{3}\left(\rho_{\mathrm{G}}-\rho_{\mathrm{L}}\right) g .
$$

Finally, the contribution to [20] from the first term of [21b] is written as the divergence of a (stress) tensor. In order to do so, note that the fluid impulse associated with a bubble $k$, viz. $\mathbf{I}_{\mathbf{L}_{k}}$, is a function of the relative bubble positions $\mathbf{x}_{i}-\mathbf{x}_{j}, i \neq j=1, \ldots, N$. Assuming that it is possible to rewrite the first term of $[21 b]$ in a pairwise decomposed form, i.e.

$$
\sum_{k=1}^{N}\left(\frac{1}{2} \dot{\mathbf{x}}_{k} \cdot \mathbf{I}_{\mathbf{L}_{k}}\right)=\sum_{i \neq j=1}^{N}\left(\sum_{k=1}^{N} \frac{1}{2} \dot{\mathbf{x}}_{k} \cdot \mathbf{I}_{\mathbf{L}_{k}}\right)_{i j},
$$

where $(\cdots)_{l j}$ is a function of $\mathbf{x}_{i}-\mathbf{x}_{j}$ only, it follows after some manipulation that the contribution under consideration is 


$$
\begin{aligned}
\sum_{j=1}^{N} \nabla_{\mathbf{x}_{j}}\left(\sum_{k=1}^{N} \dot{\mathbf{x}}_{k} \cdot \mathbf{I}_{\mathrm{L}_{k}}\right) \delta\left(\mathbf{x}_{j}-\mathbf{x}\right) \\
=\frac{1}{2} \sum_{i \neq j=1}^{N} \sum_{\mathbf{x}_{j}}\left(\sum_{k=1}^{N} \frac{1}{2} \dot{\mathbf{x}}_{k} \cdot \mathbf{I}_{\mathbf{L}_{k}}\right)_{i j}\left(\delta\left(\mathbf{x}_{j}-\mathbf{x}\right)-\delta\left(\mathbf{x}_{i}-\mathbf{x}\right)\right) \\
=\sum_{i \neq j=1}^{N} \nabla_{\mathbf{x}_{j}}\left(\frac{1}{4} \sum_{k=1}^{N} \dot{\mathbf{x}}_{k} \cdot \mathbf{I}_{\mathbf{L}_{k}}\right)_{i j}\left(\mathbf{x}_{j}-\mathbf{x}_{i}\right) \nabla_{\mathbf{x}_{j}} \delta\left(\mathbf{x}_{j}-\mathbf{x}\right) \\
=-\nabla_{\mathbf{x}} \cdot\left(\sum_{i \neq j=1}^{N} \sum_{\mathbf{x}_{j}}\left(\frac{1}{4} \sum_{k=1}^{N} \dot{\mathbf{x}}_{k} \cdot \mathbf{I}_{\mathrm{L}_{k}}\right)\left(\mathbf{x}_{j}-\mathbf{x}_{i}\right) \delta\left(\mathbf{x}_{j}-\mathbf{x}\right)\right) .
\end{aligned}
$$

Here we have used in the second step

$$
\delta\left(\mathbf{x}_{i}-\mathbf{x}\right)=\delta\left(\mathbf{x}_{j}+\left(\mathbf{x}_{i}-\mathbf{x}_{j}\right)-\mathbf{x}\right)=\delta\left(\mathbf{x}_{j}-\mathbf{x}\right)+\left(\mathbf{x}_{i}-\mathbf{x}_{j}\right) \cdot \nabla_{\mathbf{x}_{j}} \delta\left(\mathbf{x}_{j}-\mathbf{x}\right)+\cdots .
$$

Upon introducing [21a, b] and [22] into [20], and a subsequent rearrangement of terms, the conservation equation of mean Kelvin impulse (or of mean bubble momentum) becomes

$$
\frac{\partial}{\partial t}\left(n\left(\frac{4}{3} \pi a^{3} \rho_{\mathrm{G}} \mathbf{v}+\overline{\mathbf{I}}_{\mathrm{L}}\right)\right)+\nabla_{\mathbf{x}} \cdot\left(n\left(\frac{4}{3} \pi a^{3} \rho_{\mathrm{G}} \mathbf{v}+\overline{\mathbf{I}}_{\mathrm{L}}\right) \mathbf{v}\right)-\nabla_{\mathbf{x}} \cdot\left(\Theta_{\mathrm{G}}+\Xi_{\mathrm{L}}\right)=n \overline{\mathbf{F}}_{\mathrm{f}}+n \frac{4}{3} \pi a^{3}\left(\rho_{\mathrm{G}}-\rho_{\mathrm{L}}\right) \boldsymbol{g},
$$

where the flow parameters are defined by the ensemble averages:

- mean fluid impulse $\overline{\mathbf{I}}_{\mathrm{L}}$

$$
n \overline{\mathbf{I}}_{\mathrm{L}}(\mathbf{x}, t)=\frac{1}{N !} \iint \sum_{k=1}^{N}\left(-\rho_{\mathrm{L}} \int \phi \mathrm{d} \mathbf{A}_{k}\right) \delta\left(\mathbf{x}_{k}-\mathbf{x}\right) f^{N}\left(\mathscr{C}_{N}, \dot{\mathscr{C}}_{N}, t\right) \mathrm{d} \mathscr{C}_{N} \mathrm{~d} \dot{\mathscr{C}}_{N}
$$

-mean viscous drag force $\overline{\mathbf{F}}_{\mathrm{f}}$

$$
n \overline{\mathbf{F}}_{\mathrm{f}}(\mathbf{x}, t)=\frac{1}{N !} \iint \sum_{k=1}^{N} \mathbf{F}_{\mathrm{f}_{k}} \delta\left(\mathbf{x}_{k}-\mathbf{x}\right) f^{N}\left(\mathscr{C}_{N}, \dot{\mathscr{C}}_{N}, t\right) \mathrm{d} \mathscr{C}_{N}, \mathrm{~d} \dot{\mathscr{C}}_{N},
$$

with an approximate expression for the frictional force on a bubble $k$ given by [11], viz.

$$
\mathbf{F}_{\mathrm{f}_{k}}=\nabla_{\dot{\mathrm{x}}_{k}}\left(\sum_{j=1}^{N}-\frac{1}{2} \mu_{\mathrm{L}} \int \mathrm{d} \mathbf{A}_{k} \cdot \nabla\left(\frac{\partial \phi}{\partial x_{i}}\right)^{2}\right), \quad i=1,2,3, ;
$$

-(kinetic) bubble stress $\mathfrak{\Xi}_{\mathrm{G}}$

$$
\left.\Xi_{\mathrm{G}} \mathbf{x}, t\right)=-\frac{1}{N !} \iint \sum_{k=1}^{N} \frac{4}{3} \pi a^{3} \rho_{\mathrm{G}}\left(\dot{\mathbf{x}}_{k}-\mathbf{v}\right)\left(\dot{\mathbf{x}}_{k}-\mathbf{v}\right) \delta\left(\mathbf{x}_{k}-\mathbf{x}\right) f^{N}\left(\mathscr{C}_{N}, \dot{\mathscr{C}}_{N}, t\right) \mathrm{d} \mathscr{C}_{N} \mathrm{~d} \dot{\mathscr{C}}_{N},
$$

similarly defined as the kinetic contribution to stress tensor of a fluid;

- fluid impluse stress $\mathfrak{\Xi}_{\mathrm{L}}$

$$
\begin{gathered}
\Xi_{\mathrm{L}}(\mathbf{x}, t)=\Xi_{\mathrm{LK}}(\mathbf{x}, t)+\Xi_{\mathrm{LV}}(\mathbf{x}, t), \\
\Xi_{\mathrm{LK}}(\mathbf{x}, t)=-\frac{1}{N !} \iint \sum_{k=1}^{N}\left(\mathbf{I}_{\mathrm{L}_{k}}-\mathbf{I}_{\mathrm{L}}\right)\left(\dot{\mathbf{x}}_{k}-\mathbf{v}\right) \delta\left(\mathbf{x}_{k}-\mathbf{x}\right) f^{N}\left(\mathscr{C}_{N}, \dot{\mathscr{C}}_{N}, t\right) \mathrm{d} \mathscr{C}_{N} \mathrm{~d} \dot{\mathscr{C}}_{N}
\end{gathered}
$$

and

$$
\Xi_{\mathrm{LV}}(\mathbf{x}, t)=-\frac{1}{N !} \iint \sum_{i \neq j=1}^{N} \nabla_{\mathbf{x}_{j}}\left(\frac{1}{4} \sum_{k=1}^{N} \dot{\mathbf{x}}_{k} \cdot \mathbf{I}_{\mathrm{L}_{k}}\right)\left(\mathbf{x}_{j}-\mathbf{x}_{i}\right) \delta\left(\mathbf{x}_{j}-\mathbf{x}\right) f^{N}\left(\mathscr{C}_{N}, \mathrm{C}_{N} \cdot t\right) \mathrm{d} \mathscr{C}_{N} \mathrm{~d} \dot{\mathscr{C}}_{N} .
$$

As for a fluid, the fluid impulse stress tensor may be divided into a "kinetic" contribution $\mathfrak{S}_{\mathrm{LK}}$ and an "interaction potential" contribution $\varsigma_{L V}$.

Without the contributions due to the fluid, [23] is similar to the momentum equation of a gas. The presence of the fluid firstly yields a frictional drag and a buoyancy force. The irrotational pressure distribution at the bubble surface effectively increases the mean bubble momentum; in addition it gives rise to a stress. This can be explained in a similar manner to the occurrence of a stress in an ordinary fluid. Consider a "particle" of bubbles moving along with the mean velocity 
of the bubbles. A force should be exerted by the "material" outside the particle in order to prevent bubbles from entering or leaving the particle as a consequence of their velocity fluctuations, i.e. in order to prevent a change in the "additional momentum" of the particle of bubbles. Part of the force is associated with the transient pressure distribution in the fluid, and related to the mean square of the fluctuations of the fluid impulse; the other part is needed to overcome the attractive and repulsive forces between the bubbles due to the dynamic pressure distribution at their surfaces. The force can be interpreted as a contact force exerted across the surface surrounding the particle; it will be proportional to the size of the surface element on which it acts and can therefore be associated with an effective stress.

To describe the void fraction wave dynamics, [23] should be supplemented by relations that express the above defined flow parameters in terms of $\epsilon, \mathbf{v}$ and their derivatives. It will be clear that it is very difficult to give a rigorous derivation of these relations by means of solving the Liouville equation or a contracted version of it, and at this point we therefore have to change to phenomenological reasoning. Even this is difficult, because virtually no information is available on the dynamics of the bubble interactions, and the associated statistics. Nevertheless, the above analysis has revealed the way in which the pressure forces on the bubbles, arising from the irrotational part of the fluid motion, are to be included in the bubble momentum equation; this, and the analogy with the kinetic theory of gases, give some suggestions how to proceed with the modelling of the flow parameters.

\section{MODELLING OF THE ONE-DIMENSIONAL EQUATIONS OF MOTION}

In a uniform flow the mean velocity of the bubbles is determined by the balance between the buoyancy force and the mean viscous drag force exerted on the bubbles; [23] takes the form

$$
n \bar{F}_{\mathrm{f}}=n\left(\frac{4}{3} \pi a^{3}\right)\left(\rho_{\mathrm{G}}-\rho_{\mathrm{L}}\right) g .
$$

The effect of hydrodynamic interactions between the bubbles on the mean frictional force may be represented by introducing a function $f_{0}(\epsilon)$, which takes on a value of 1 in the limit of vanishing void fraction. The subscript 0 refers to the uniform state. In combination with the expression for the drag force on a single bubble [1], [24] becomes

$$
-12 \pi a \mu_{\mathrm{L}} f_{0}(\epsilon) v_{0}(\epsilon)=\frac{4}{3} \pi a^{3}\left(\rho_{\mathrm{G}}-\rho_{\mathrm{L}}\right) g
$$

and the mean velocity of rise, with respect to a zero volume flux frame, is given by

$$
v_{0}(\epsilon)=\frac{v_{\infty}}{f_{0}(\epsilon)},
$$

where $v_{x}$, the velocity of a single bubble, is determined by [2]. Experiments suggest that

$$
v_{0}(\epsilon)=v_{x}(1-\epsilon)^{p}
$$

with an exponent $p$ within the range 1.8-2.3 (Hetsroni 1982, section 2.3). In the calculations of the following sections we shall use $p=2$, which corresponds to Wallis' (1969) expression for the mean velocity of rise in a stagnant liquid (the slip velocity), viz $U_{\mathrm{G} 0}(\epsilon)=v_{\infty}(1-\epsilon)$. Relationships for the mean fluid impulse and the stresses may be postulated as well for this uniform flow. The fluid impulse is written as

$$
n \bar{I}_{\mathrm{L}}=n\left(\frac{2}{3} \pi a^{3} \rho_{\mathrm{L}}\right) m_{0}(\epsilon) v_{0}(\epsilon),
$$

where $2 / 3 \pi a^{3} \rho_{\mathrm{L}}$ is the added mass of a single sphere. The function $m_{0}(\epsilon)$ again represents the effect of hydrodynamic interactions and $m_{0}(\epsilon) \rightarrow 1$ for $\epsilon \rightarrow 0$. Biesheuvel \& Spoelstra (1989) examined various expressions for $m_{0}(\epsilon)$ given in the two-phase flow literature; it was found that

$$
m_{0}(\epsilon)=\frac{1+2 \epsilon}{1-\epsilon}
$$


due to Zuber (1964), gives reliable results up to large values of the void fraction. In a uniform flow the role of the stresses $\Xi_{G}$ and $\Xi_{L}$ is similar to that of a pressure. The "kinetic" contribution to the effective pressure is likely to be of the form

$$
p_{\mathrm{cK}}(\epsilon)=n\left[\frac{4}{3} \pi a^{3} \rho_{\mathrm{G}}+\frac{2}{3} \pi a^{3} \rho_{\mathrm{L}} m_{0}(\epsilon)\right] \overline{\Delta v^{2}},
$$

i.e. proportional to the effective density $\epsilon\left(\rho_{\mathrm{G}}+1 / 2 \rho_{\mathrm{L}} m_{0}(\epsilon)\right)$ of the bubbles and to the mean square of their velocity fluctuations. A measure of these velocity fluctuations is the mean velocity of the bubbles and Batchelor (1988), therefore, proposed writing

$$
\overline{\Delta v^{2}}(\epsilon)=H(\epsilon) v_{0}^{2}(\epsilon)
$$

with

$$
H(\epsilon) \approx \frac{\epsilon}{\epsilon_{\mathrm{cp}}}\left(1-\frac{\epsilon}{\epsilon_{\mathrm{cp}}}\right)
$$

The latter choice meets requirements of vanishing of the velocity fluctuations in the limit $\epsilon \rightarrow 0$ and in the limit of closest packing, $\epsilon \rightarrow \epsilon_{\mathrm{cp}}$. An appropriate value of $\epsilon_{\mathrm{cp}}$ is 0.62 . It is not clear how to model the "potential" contribution to the effective pressure. Calculations by Biesheuvel \& van Wijngaarden (1982) for the case of two spherical bubbles indicate that for angles between the separation vector and the vector of gravity larger than about $35^{\circ}$ the interaction force between the bubbles is attractive for all sphere separations, and that it is repulsive for smaller angles. This suggests that the "potential" contribution to the effective pressure will be negative. For small values of the void fraction one would expect that it is small compared to that related to the velocity fluctuations of the bubbles; near closest packing the "potential" contribution is likely to be dominant. In the following we will tentatively ignore the "potential stress"; the subscript $\mathrm{K}$ will be dropped.

A first step towards a modelling of non-uniform flows is to assume the dependency on the local value of the void fraction of the viscous drag and fluid impulse to remain unaltered when the flow is slightly perturbed. Merely replacing $v_{0}(\epsilon)$ in [25] and [28] by the local value of the mean bubble velocity yields

$$
n \bar{F}_{\mathrm{f}}=n\left[-12 \pi a \mu_{\mathrm{L}} f_{0}(c) v\right]
$$

and

$$
n \bar{I}_{\mathrm{L}}=n\left(\frac{2}{3} \pi a^{3} \rho_{\mathrm{L}}\right) m_{0}(\epsilon) v .
$$

The fact that the bubbles are in "random motion" in the presence of a void fraction gradient may have an important diffusive effect on the wave propagation that needs to be taken into account: this fluctuating motion will give rise to a diffusive flux of bubble number density equal to

$$
-\delta_{\mathrm{e}}(n) \frac{\partial n}{\partial z}
$$

where $\delta_{\mathrm{e}}$ is an effective diffusivity. The corresponding change in the mean bubble velocity is

$$
-\frac{\delta_{\mathrm{e}}(n)}{n} \frac{\partial n}{\partial z}
$$

It is as if each of the bubbles is acted upon by a steady force given by

$$
-12 \pi a \mu_{\mathrm{L}} f_{0}(\epsilon) \frac{\delta_{\mathrm{e}}(\epsilon)}{\epsilon} \frac{\partial \epsilon}{\partial z}
$$

The mean frictional force will therefore be written as

$$
n \bar{F}_{\mathrm{f}}=\frac{12 \pi a \mu_{\mathrm{L}}}{\frac{4}{3} \pi a^{3}} f_{0}(\epsilon) \epsilon\left[v+\frac{\delta_{\varepsilon}(\epsilon)}{\epsilon} \frac{\partial \epsilon}{\partial z}\right]
$$


If we assume that in a non-uniform bubbly fluid it is primarily the non-uniformity in the mean bubble velocity that causes an additional transfer of bubble momentum and fluid impulse associated with a stress we may postulate that the stress will give rise to a force

$$
\frac{\partial}{\partial z}\left(-p_{\mathrm{e}}(\epsilon)+\epsilon\left[\rho_{\mathrm{G}}+\frac{1}{2} \rho_{\mathrm{L}} m_{0}(\epsilon)\right] v_{\mathrm{e}}(\epsilon) \frac{\partial v}{\partial z}\right)
$$

The physical processes that underlie the effective diffusivities $\delta_{\mathrm{e}}$ and $v_{\mathrm{e}}$ suggest that these parameters should be proportional to a length scale and a velocity scale of the fluctuating bubble motion. We shall follow Batchelor (1988) and choose

$$
\delta_{\mathrm{e}}(\epsilon)=\alpha a\left[\overline{\Delta v^{2}}(\epsilon)\right]^{1 / 2}=\alpha a v_{0}(\epsilon)[H(\epsilon)]^{1 / 2}
$$

and

$$
\nu_{\mathrm{e}}(\epsilon)=\beta a\left[\overline{\Delta v^{2}}(\epsilon)\right]^{1 / 2}=\beta a v_{0}(\epsilon)[H(\epsilon)]^{1 / 2}
$$

with $\alpha$ and $\beta$ constants of $O(1)$; in the calculations of sections 6 and $7 \alpha$ and $\beta$ will be set equal to 1 .

On the basis of the above proposals the one-dimensional equations of motion become

$$
\frac{\partial \epsilon}{\partial t}+\frac{\partial}{\partial z} \epsilon v=0
$$

and

$$
\begin{aligned}
\frac{\partial}{\partial t}\left(\epsilon\left(\rho_{\mathrm{G}}+\frac{1}{2} \rho_{\mathrm{L}} m_{0}\right) v\right)+\frac{\partial}{\partial z}\left(\epsilon\left(\rho_{\mathrm{G}}+\frac{1}{2} \rho_{\mathrm{L}} m_{0}\right) v^{2}\right)-\frac{\partial}{\partial z} & \left(-p_{\mathrm{e}}+\mu_{\mathrm{e}} \frac{\partial v}{\partial z}\right) \\
& =-\frac{12 \pi a \mu_{\mathrm{L}}}{\frac{4}{3} \pi a^{3}} \epsilon f_{0}\left(v+\frac{\delta_{\mathrm{e}}}{\epsilon} \frac{\partial \epsilon}{\partial z}\right)-\epsilon\left(\rho_{\mathrm{G}}-\rho_{\mathrm{l}}\right) g
\end{aligned}
$$

where we have introduced for convenience

$$
\mu_{\mathrm{e}}(\epsilon)=\epsilon\left[\rho_{\mathrm{G}}+\frac{1}{2} \rho_{\mathrm{L}} m_{0}(\epsilon)\right] v_{\mathrm{E}}(\epsilon) .
$$

Functional relationships for $f_{0}, m_{0}, p_{\mathrm{e}}, \delta_{\mathrm{e}}$ and $\mu_{\mathrm{e}}$ are given by [26], [27] and [30]-[33].

It is useful to note that upon use of [25] and [34] the momentum conservation equation [35] can be rewritten as a relaxation equation

$$
\begin{aligned}
\left(\frac{\partial}{\partial t}+v \frac{\partial}{\partial z}\right) v-\frac{\frac{1}{2} \rho_{\mathrm{L}} m_{0}^{\prime} \epsilon}{\rho_{\mathrm{G}}+\frac{1}{2} \rho_{\mathrm{L}} m_{0}} v \frac{\partial v}{\partial z}-\frac{1}{\epsilon\left(\rho_{\mathrm{G}}+\frac{1}{2} \rho_{\mathrm{L}} m_{0}\right)}\left[\frac{\partial}{\partial z}\left(-p_{\mathrm{e}}+\mu_{\mathrm{e}} \frac{\partial v}{\partial z}\right)\right] & =-\frac{1}{\tau_{\mathrm{e}}}\left(v-v_{0}+\frac{\delta_{\mathrm{e}}}{\epsilon} \frac{\partial \epsilon}{\partial z}\right) .
\end{aligned}
$$

The prime denotes differentiation with respect to $\epsilon$ and $\tau_{\mathrm{e}}$ is an effective relaxation time given by

$$
\tau_{\mathrm{e}}(\epsilon)=\frac{\left[\rho_{\mathrm{G}}+\frac{1}{2} \rho_{\mathrm{L}} m_{0}(\epsilon)\right] a^{2}}{9 \mu_{\mathrm{L}} f_{0}(\epsilon)}
$$

This shows that the mean motion of the bubbles in a perturbed flow is characterized by a relaxation process: frictional forces tend to adapt the mean bubble velocity to a velocity that corresponds to a uniform state with local value of the void fraction, the inertia of the gas bubbles and the fluid oppose this tendency.

Equations [34] and [35] can be used to describe the propagation of a localized void fraction disturbance to a uniform bubbly fluid; in that case the mean volume flux of the dispersion remains constant. In the experiments of Boure and co-workers the void fraction waves are caused by changes in the supply of one or both of the phases, the mean volume flux then being a function of time. By making use of the theory for an accelerating body in a uniformly accelerating perfect fluid (Batchelor 1967, p. 409), [34] and [35] can be adapted to describe this situation. It is more 
convenient to use velocities that are defined with respect to a laboratory frame. If the mean bubble velocity and the mean velocity of the fluid in this reference frame are denoted by $U_{\mathrm{G}}$ and $U_{\mathrm{L}}$, the mean velocity of the dispersion is given by

$$
U=\epsilon U_{\mathrm{G}}+(1-\epsilon) U_{\mathrm{L}} .
$$

The pertinent conservation equations are

$$
\begin{aligned}
\frac{\partial \epsilon}{\partial t}+\frac{\partial}{\partial z} \epsilon U_{\mathrm{G}} & =0 \\
\frac{\partial U}{\partial z} & =0
\end{aligned}
$$

and

$$
\begin{aligned}
\frac{\partial}{\partial t}(\epsilon & {\left.\left[\rho_{\mathrm{G}} U_{\mathrm{G}}+\frac{1}{2} \rho_{\mathrm{L}} m_{0}\left(U_{\mathrm{G}}-U\right)\right]\right)+\frac{\partial}{\partial z}\left(\epsilon\left[\rho_{\mathrm{G}} U_{\mathrm{G}}+\frac{1}{2} \rho_{\mathrm{L}} m_{0}\left(U_{\mathrm{G}}-U\right)\right] U_{\mathrm{G}}\right) } \\
& -\frac{\partial}{\partial z}\left(-p_{\mathrm{e}}+\mu_{\mathrm{e}} \frac{\partial U_{\mathrm{G}}}{\partial z}\right)-\epsilon \rho_{\mathrm{L}} \frac{\partial U}{\partial t} \\
= & -\frac{12 \pi a \mu_{\mathrm{L}}}{\frac{4}{3} \pi a^{3}} \epsilon f_{0}\left[\left(U_{\mathrm{G}}-U\right)+\frac{\delta_{\mathrm{e}}}{\epsilon} \frac{\partial \epsilon}{\partial z}\right]-\epsilon\left(\rho_{\mathrm{G}}-\rho_{\mathrm{L}}\right) g .
\end{aligned}
$$

The above equations and constitutive relations for the flow parameters have been modelled under the assumptions that the dispersion consists of spherical bubbles and that the flow around the bubbles is laminar. In most of the experiments of the group at Grenoble these conditions are not met; the bubbles have ellipsoidal shape and the fluid flow is turbulent. The fluid turbulence may be considered to act like a random forcing on the bubbles, that enhances their fluctuating motion. The momentum equation [41] can still be used in this case, but the constitutive relations for the bubble pressure and the effective diffusivities may need a slight modification. At low void fractions in particular, the mean square of the velocity fluctuations will be larger than in the case of laminar flow. For bubbles of ellipsoidal shape other functional relationships for the mean Kelvin impulse and the mean frictional force as well as for the velocity fluctuations may be appropriate. Thus, it can not be expected that the model derived in this section will give quantitative agreement with the observations of Boure's group; nevertheless, it should be possible to bring out the observed features of the propagation of void fraction waves and the (in)stability of bubbly flows.

\section{LINEAR VOID FRACTION WAVES}

Let the uniform state by given by $\epsilon=\epsilon_{0}, U=U_{0}$ and $U_{\mathrm{G}}=U_{\mathrm{G}_{0}}\left[=U_{0}+v_{0}\left(\epsilon_{0}\right)\right]$. Linearization of [39]-[41] yields the wave-hierarchy equation

$$
\tau_{\mathrm{e}}\left[\left(\frac{\partial}{\partial t}+c^{+} \frac{\partial}{\partial z}\right)\left(\frac{\partial}{\partial t}+c^{-} \frac{\partial}{\partial z}\right) \epsilon-v_{\mathrm{e}}\left(\frac{\partial}{\partial t}+U_{\mathrm{C}_{0}} \frac{\partial}{\partial z}\right) \frac{\partial^{2} \epsilon}{\partial z^{2}}\right]+\left[\left(\frac{\partial}{\partial t}+c_{0} \frac{\partial}{\partial z}\right) \epsilon-\delta_{\mathrm{e}} \frac{\partial^{2} \epsilon}{\partial z^{2}}\right]=0
$$

with lower-order and higher-order wave velocities given by

$$
c_{0}=U_{\mathrm{G}_{0}}+\epsilon_{0} v_{0}^{\prime}
$$

and

$$
c^{ \pm}=U_{\mathrm{G}_{0}}-\frac{\frac{1}{4} \epsilon_{0} \rho_{\mathrm{L}} m_{0}^{\prime} v_{0}}{\rho_{\mathrm{G}}+\frac{1}{2} \rho_{\mathrm{L}} m_{0}} \pm\left[\left(\frac{\frac{1}{4} \epsilon_{0} \rho_{\mathrm{L}} m_{0}^{\prime} v_{0}}{\rho_{\mathrm{G}}+\frac{1}{2} \rho_{\mathrm{L}} m_{0}}\right)^{2}+\frac{p_{\mathrm{e}}^{\prime}}{\rho_{\mathrm{G}}+\frac{1}{2} \rho_{\mathrm{L}} m_{0}}\right]^{1 / 2}
$$

Again a prime denotes differentiation with respect to $\epsilon$ and it is understood that functions of the void fraction are evaluated at the unperturbed state, $\epsilon=\epsilon_{0}$. Wave motion governed by a wave-hierarchy equation like [42] appears in a large variety of fluid mechanical systems: flood surges in rivers (Lighthill \& Whitman 1955), waves on turbulent liquid films, sediment waves on river beds (Kluwick 1977), voidage waves in fluidized beds (Liu 1982, 1983) and sedimentation 
waves (Kluwick 1983). The wave motion is usually discussed in terms of the interaction between kinematic and dynamic waves. The theory originates from Lighthill \& Whitman, a detailed analysis may be found in Whitman $(1959,1974)$.

The lower-order wave velocity $c_{0}$ corresponds to that of the kinematic wave approximation, in which one sets $\tau_{\mathrm{e}}=v_{\mathrm{e}}=0$. This means that is assumed that the characteristic length and time scales of the disturbances are sufficiently large to neglect relaxation effects due to the inertia of the bubbles and the fluid, and diffusive effects associated with the random bubble motion. Equation (40) then reduces to a functional relationship between the local volumetric flux of bubbles and the local bubble volume density

$$
\epsilon U_{\mathrm{G}}=\epsilon\left[U+v_{0}(\epsilon)\right]
$$

A similar relationship forms the basis of Kynch's (1952) analysis of sedimentation waves in suspensions of small particles, that introduced the theory of kinematic waves. Linearization of [39] and [40] with [45] yields the wave equation

$$
\frac{\partial \epsilon}{\partial t}+\left(U+v_{0}+\epsilon \frac{\mathrm{d}}{\mathrm{d} \epsilon} v_{0}\right)_{\epsilon=\varsigma_{0}} \frac{\partial \epsilon}{\partial z}=0
$$

with a wave velocity equal to [43]. Alternatively, in the dynamic wave approximation, i.e. $\tau_{\mathrm{e}} \rightarrow \infty$, one assumes that inertia effects dominate the bubble motion. This would be appropriate for sufficiently small characteristic length or time scales of the disturbances. Equations [39]-[41] are then very similar to the one-dimensional equations of gas dynamics. The higher-order wave velocities [44] are the linearized characteristics; as in gas dynamics, dissipation occurs due to random motions of the constituent particles. For a dispersion of bubbles with radii of $0.4 \mathrm{~mm}, \mathrm{a}$ value used in all subsequent calculations, the relaxation time $\tau_{\mathrm{e}}$ is of order $10^{-2} \mathrm{~s}$ and the diffusivities $\delta_{\mathrm{e}}$ and $v_{\mathrm{e}}$ are of order $10^{-3} \mathrm{~m}^{2} / \mathrm{s}$. Although these parameters are small, the experimental results can only be explained by considering how the inertia of the two phases and the random bubble motion, that are related to these parameters, affect the kinematic wave motion. It should be noted that in the context of bubbly flows the use of the names kinematic and dynamic waves, introduced by Lighthill \& Whitman, may be misleading since the latter usually refers to the propagation of pressure disturbances. It is amusing that sound waves in a bubbly fluid are also affected by a relaxation process (Noordzij \& van Wijngaarden 1974).

If periodic disturbances are maintained at the pipe inlet, the features of the void fraction waves may be summarized as follows:

For relatively low radial frequencies the wave propagation is approximately described by a linearized Burgers/Korteweg-de Vries equation:

$$
\frac{\partial \epsilon}{\partial t}+c_{0} \frac{\partial \epsilon}{\partial z} \approx\left[\tau_{\mathrm{e}}\left(c^{+}-c_{0}\right)\left(c_{0}-c^{-}\right)+\delta_{\mathrm{e}}\right] \frac{\partial^{2} \epsilon}{\partial z^{2}}+\tau_{\mathrm{e}} v_{\mathrm{e}}\left(U_{\mathrm{G}_{0}}-c_{0}\right) \frac{\partial^{3} \epsilon}{\partial z^{3}},
$$

which can be obtained from [42] by using $\partial / \partial t \approx-c_{0} \partial / \partial z$ in all derivatives except that of the lower-order wave. The solution is

$$
\epsilon \propto \exp (\gamma z-i \omega t)
$$

with

$$
\gamma \approx \frac{i \omega}{c_{0}}\left[1-\frac{v_{\mathrm{e}} \tau_{\mathrm{e}} \omega^{2}\left(U_{\mathrm{G}_{0}}-c_{0}\right)}{c_{0}^{3}}\right]-\frac{\omega^{2}}{c_{0}^{3}}\left[\tau_{\mathrm{e}}\left(c^{+}-c_{0}\right)\left(c_{0}-c^{-}\right)+\delta_{\mathrm{e}}\right] .
$$

The phase velocity shows a slight dependency on frequency. That this dependency can hardly be noticed experimentally becomes clear from an evaluation of the term $v_{\mathrm{e}} \tau_{\mathrm{e}} \omega^{2}\left(U_{0}+v_{0}-c_{0}\right) / c_{0}^{3}$ for the case in which there is no mean fluid motion under unperturbed conditions, i.e. when $U_{\mathrm{G}_{0}}=v_{0} /\left(1-\epsilon_{0}\right)$. This yields $3 \cdot 10^{-6} \omega^{2}$ and $2 \cdot 10^{-8} \omega^{2}$ for $\epsilon_{0}=0.1$ and $\epsilon_{0}=0.2$, respectively. Both quantities are much smaller than 1 in the range of frequencies for which [4] is applicable $\left(\omega \ll 1 / \tau_{e}\right.$, i.e. $\omega / 2 \pi \ll 16 \mathrm{~Hz}$ ). Also in agreement with the experimental observations is that the phase velocity is less than the gas velocity and larger than the fluid velocity. The first conclusion follows directly 
from [43], by noting that the mean bubble velocity decreases which increasing void fraction. To reach the second conclusion, use [26] and [38] to write [43] as

$$
c_{0}=U_{\mathrm{L}_{0}}+\left(U_{\mathrm{G}_{0}}-U_{\mathrm{L}_{0}}\right)\left[1-\frac{\epsilon_{0}\left(1-\epsilon_{0}\right) f_{0}^{\prime}}{f_{0}}\right] .
$$

Both terms between parentheses are easily seen to be positive in the void fraction range used in the experiments.

Figure 1 shows the gain factor $\exp \left[\operatorname{Re}(\gamma) \omega^{2} \Delta z\right]$ over a distance $\Delta z=20 \mathrm{~cm}$, the distance between the impedance probes in the experiments of Mercadier (1981), for imposed frequencies of 0.5, 1.0, $1.5,2.0$ and $2.5 \mathrm{~Hz}$; the liquid is taken to be stagnant. For $\epsilon_{0}<0.30$ the attenuation increases with increasing value of the void fraction, which clearly contradicts the conclusions of Mercadier. For values of $\epsilon_{0}$ between 0.30 and 0.35 the attenuation rate drops significantly while for $\epsilon_{0}$ larger than about 0.35 the disturbances are amplified, indicating that the uniform flow is unstable. It will be clear from the figures that, for $0.10 \leqslant \epsilon_{0} \leqslant 0.30$, waves with frequencies higher than only a few $\mathrm{Hz}$ will be hard to detect, particularly with signal probes mounted some distance above the pipe inlet. This will be easier when a liquid flux is supplied to the system in order to increase the velocity of the waves. Also higher frequencies will be detected. As an example, figure 2 shows the gain factor along the tube of waves with frequencies $2,4,6,8$ and $10 \mathrm{~Hz}$ in a bubbly fluid with mean void fraction of $15 \%$ and a superficial liquid velocity of $0.18 \mathrm{~m} / \mathrm{s}$, the value used in the experiments of Matuszkiewicz et al. (1987).

Next, consider the propagation of periodic disturbances of relatively high frequency. To this end it is useful to incorporate $\delta_{\mathrm{e}} \partial^{2} / \partial z^{2}$ in the higher-order wave operators. The wave equation then becomes

$$
\tau_{\mathrm{e}}\left(\frac{\partial}{\partial t}+c_{+} \frac{\partial}{\partial z}\right)\left(\frac{\partial}{\partial t}+c_{-} \frac{\partial}{\partial z}\right) \epsilon+\left(\frac{\partial}{\partial t}+c_{0} \frac{\partial}{\partial z}\right) \epsilon=v_{\mathrm{e}} \tau_{\mathrm{e}}\left(\frac{\partial}{\partial t}+U_{\mathrm{G}_{0}} \frac{\partial}{\partial z}\right) \frac{\partial^{2} \epsilon}{\partial z^{2}}
$$

with higher-order wave velocities [44] modified into

$$
c_{ \pm}=U_{\mathrm{G}_{0}}-\frac{\frac{1}{4} \epsilon_{0} \rho_{\mathrm{L}} m_{0}^{\prime} v_{0}}{\rho_{\mathrm{G}}+\frac{1}{2} \rho_{\mathrm{L}} m_{0}} \pm\left[\left(\frac{\frac{1}{4} \epsilon_{0} \rho_{\mathrm{L}} m_{0}^{\prime} v_{0}}{\rho_{\mathrm{G}}+\frac{1}{2} \rho_{\mathrm{L}} m_{0}}\right)^{2}+\frac{p_{\mathrm{e}}^{\prime}}{\rho_{\mathrm{G}}+\frac{1}{2} \rho_{\mathrm{L}} m_{0}}+\frac{\delta_{\mathrm{e}}}{\tau_{\mathrm{e}}}\right]^{1 / 2},
$$

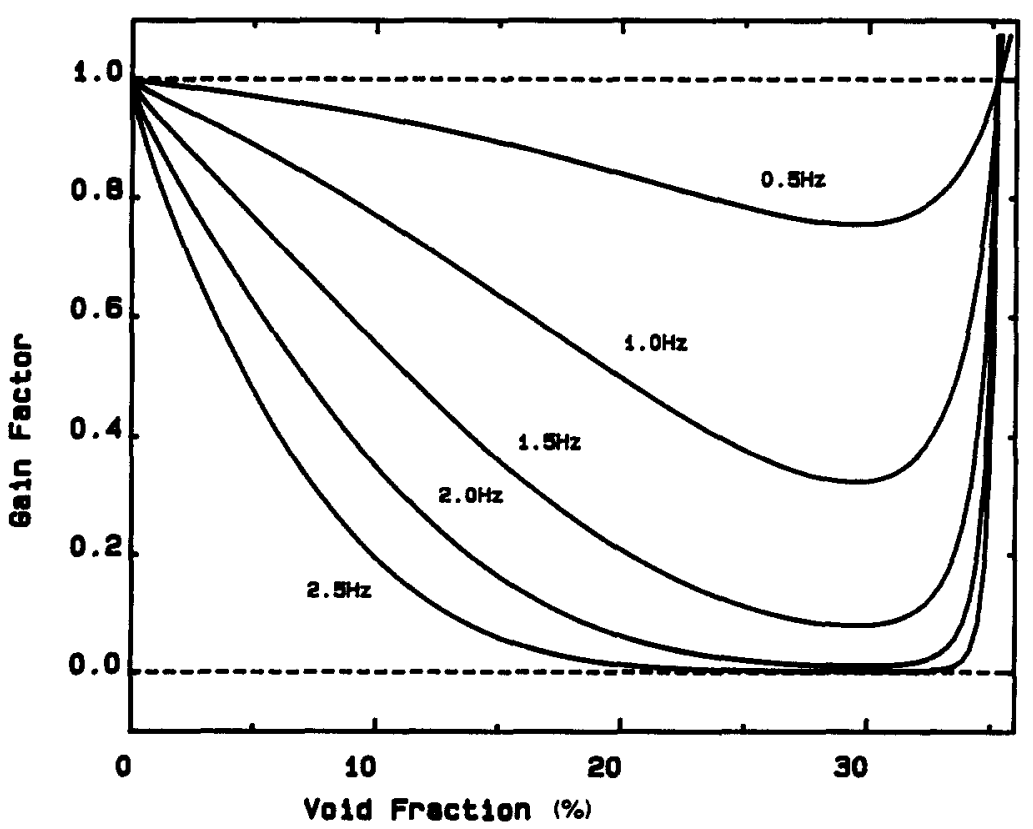

Figure 1. Gain factor over a distance of $20 \mathrm{~cm}$ as a function of the void fraction, for waves with frequencies of $0.5,1.0,1.5,2.0$ and $2.5 \mathrm{~Hz}$. The liquid is stagnant. 


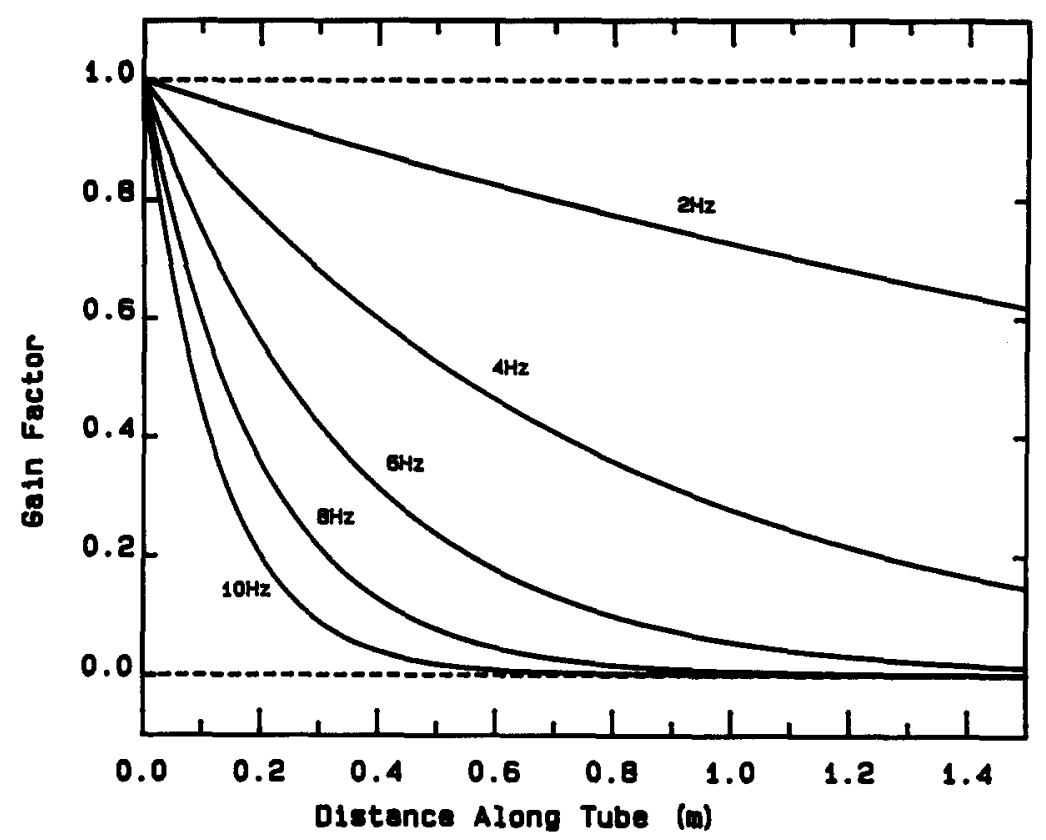

Figure 2. Gain factor along a tube of void fraction waves of frequencies of $2,4,6,8$ and $10 \mathrm{~Hz}$. The mean void fraction is $15 \%$ and the superficial liquid velocity is $0.18 \mathrm{~m} / \mathrm{s}$.

and with an unaltered lower-order wave velocity given by [43]. An approximate solution is given by

$$
\epsilon \propto \exp \left(\gamma_{ \pm} z-i \omega t\right)
$$

with

$$
\gamma_{+} \approx \frac{i \omega}{c_{+}}-\frac{1}{c_{+}}\left[\frac{c_{+}-c_{0}}{\tau_{\mathrm{e}}\left(c_{+}-c_{-}\right)}+v_{\mathrm{e}} \omega^{2} \frac{c_{+}-U_{\mathrm{G}_{0}}}{\left(c_{+}\right)^{2}\left(c_{+}-c_{-}\right)}\right]
$$

and

$$
\gamma_{-} \approx \frac{i \omega}{c_{-}}-\frac{1}{c_{-}}\left[\frac{c_{0}-c_{-}}{\tau_{e}\left(c_{+}-c_{-}\right)}+v_{\mathrm{e}} \omega^{2} \frac{U_{\mathbf{G}_{0}}-c_{-}}{\left(c_{-}\right)^{2}\left(c_{+}-c_{-}\right)}\right]
$$

Solution [51] confirms that high-frequency disturbances are much stronger attenuated than those of low frequency. As an example: evaluation shows that in a stagnant liquid and for a mean void fraction of $10 \%$ the gain factor over $20 \mathrm{~cm}$ of the $c_{+}$-wave with frequency of $20 \mathrm{~Hz}$ is about $4 \cdot 10^{-13}$ ! In contrast, figure 1 shows that in this case the gain factor of a wave with frequency of $2.0 \mathrm{~Hz}$ is about 0.94 .

It can be inferred from Whitham $(1959,1974)$ that for the general signalling problem the wave motion at times $t \gg \tau_{\mathrm{e}}$ is described by the lower-order wave equation [46]. Since $\tau_{\mathrm{e}} \simeq 0.01 \mathrm{~s}$, it is this lower-order wave motion that will be detected in an experiment.

In order to establish that the group in Grenoble did indeed record the lower-order wave motion we performed some experiments ourselves, employing the same measuring techniques. The test rig consisted of a tube of square cross-section of about $8 \times 8 \mathrm{~cm}^{2}$ with pairs of impedance probes for $5 \times 3 \mathrm{~cm}^{2}$. The axial distance between pairs of probes were $20 \mathrm{~cm}$. For simplicity stagnant fluid was used. The bubbles were produced with hypodermic needles, a method meant to reduce the scatter in the bubble sizes and shapes. This proved to give satisfactory results for a fixed value of the void fraction, but unfortunately the mean bubble diameter varied from $2.0 \mathrm{~mm}$ till about $3.5 \mathrm{~mm}$ when increasing the mean void fraction of $2 \%$ up to $16 \%$. This variation and the fact that the bubbles were not of spherical shape meant that [27] could not be used to predict the mean bubble velocity.

Measured values of the wave speed $(\diamond)$ and the mean velocity of rise of the bubbles $(O)$ are

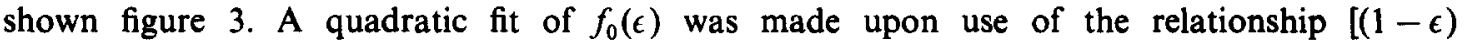
$\left.U_{\mathrm{G}}(\epsilon)\right]=v_{0}(\epsilon)=c_{\infty} /\left[f_{0}(\epsilon)\right]$, where $c_{\infty}$ is a constant. This was subsequently used to calculate the wave 


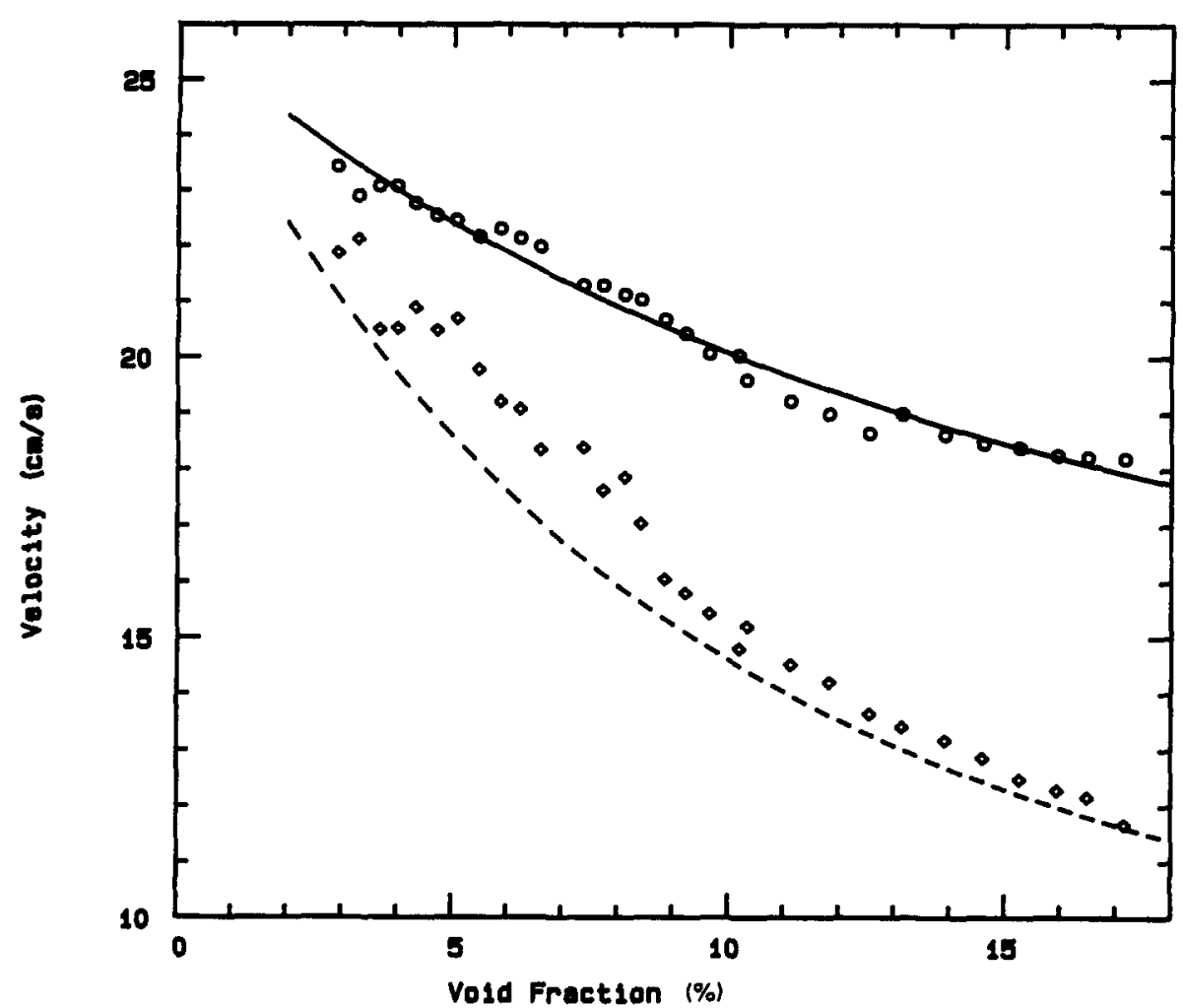

Figure 3. Measurements of the mean velocity of rise of bubbles in stagnant water $(O)$ and the velocity of propagation of naturally occurring void fraction perturbations $(\diamond), \ldots$. Prediction of the wave speed from a calculation based on a fit of the measured bubble velocities $(\stackrel{-}{-}$ ) and the relation for the kinematic wave speed.

velocity from [48] with $U_{\mathrm{L}_{0}}=0$. Comparison of the resulting curve (-- ) with the measured wave speeds $(\diamond)$ establishes in our view that these waves correspond with the lower-order waves of the theory. It turned out that with increasing void fraction longer correlation times were needed to obtain a clear peak in the cross-correlation curve, from which the wave velocity can be deduced. This means that the damping did increase with increasing void fraction; which is what the theory predicts.

\section{LINEAR STABILITY OF UNIFORM BUBBLY FLOWS}

The fact that the propagation of void fraction disturbances of infinitesimal amplitude is governed by the wave-hierarchy equation [49] provides a simple criterion for the stability of uniform bubbly flows. The work of Whitham $(1959,1974)$ shows for $v_{\mathrm{e}}=0$ that uniform flows are unstable to concentration perturbations if

$$
c_{-} \leqslant c_{0} \leqslant c_{+}
$$

is violated. In that case the attenuation rates of both the lower-order waves and the higher-order waves become negative [note that $\tau_{\mathrm{e}}\left(c^{+}-c_{0}\right)\left(c_{0}-c^{-}\right)+\delta_{\mathrm{e}}$ in [47] is equivalent to $\left.\tau_{\mathrm{e}}\left(c_{+}-c_{0}\right)\left(c_{0}-c_{-}\right)\right]$. For non-zero values of $v_{\mathrm{e}}$ this criterion remains unaltered, as can be seen by the following analysis based on Needham \& Merkin (1984) and Batchelor (1988).

Consider a spatially periodic disturbance, with wavenumber $k$, to a uniform flow. The solution to [49] is

$$
\epsilon \propto \exp i k(z-c t)
$$

with

$$
\tau_{\mathrm{e}} k\left(c_{+}-c\right)\left(c-c_{-}\right)+i k^{2} v_{\mathrm{e}} \tau_{\mathrm{e}}\left(c-U_{\mathrm{G}_{0}}\right)+i\left(c-c_{0}\right)=0 .
$$


To find the roots of the quadratic equation, first rewrite it in the form

$$
(c-\bar{c})^{2}+i P(c-\bar{c})-(Q+i R)=0,
$$

where

$$
\begin{gathered}
\bar{c}=\frac{1}{2}\left(c_{+}+c_{-}\right), \\
P=\frac{1+k^{2} v_{\mathrm{e}} \tau_{\mathrm{e}}}{k \tau_{\mathrm{e}}}, \\
Q=\frac{1}{4}\left(c_{+}-c_{-}\right)^{2}
\end{gathered}
$$

and

$$
R=\frac{\left(c_{0}-\bar{c}\right)+k^{2} v_{\mathrm{e}} \tau_{\mathrm{e}}\left(U_{\mathrm{G}_{0}}-\bar{c}\right)}{k \tau_{\mathrm{e}}} .
$$

The roots of [54] are

$$
c=\bar{c}-\frac{1}{2} i P \mp i\left(\frac{1}{4} P^{2}-Q-i R\right)^{1 / 2} .
$$

Introducing $c=c_{\mathrm{r}}+i c_{\mathrm{i}}$, where $c_{\mathrm{r}}$ is the phase velocity and $k c_{\mathrm{i}}$ is the growth rate, into [56] finally yields

$$
c_{\mathrm{r}}=\bar{c} \pm\left\{-\frac{1}{8} P^{2}+\frac{1}{2} Q+\frac{1}{2}\left[\left(\frac{1}{4} P^{2}-Q\right)^{2}+R^{2}\right]^{1 / 2}\right\}^{1 / 2}
$$

and

$$
c_{\mathrm{i}}=-\frac{1}{2} P \mp\left\{\frac{1}{8} P^{2}-\frac{1}{2} Q+\frac{1}{2}\left[\left(\frac{1}{4} P^{2}-Q\right)^{2}+R^{2}\right]^{1 / 2}\right\}^{1 / 2} .
$$

The uniform flow is stable when $c_{\mathrm{i}} \leqslant 0$. Some manipulation shows that this condition is equivalent to

$$
R^{2} \leqslant P^{2} Q
$$

or, with $[55 \mathrm{a}-\mathrm{d}]$

$$
\left[\left(c_{0}-c_{-}\right)-k^{2} v_{\mathrm{e}} \tau_{\mathrm{e}}\left(U_{\mathrm{G}_{0}}-c_{-}\right)\right]\left[\left(c_{+}-c_{0}\right)+k^{2} v_{\mathrm{e}} \tau_{\mathrm{e}}\left(c_{+}-U_{\mathrm{G}_{0}}\right)\right] \geqslant 0 .
$$

Condition [58] can only be satisfied when

$$
c_{-} \leqslant U_{\mathrm{G}_{0}} \leqslant c_{+}
$$

and

$$
c_{-} k^{2} v_{\mathrm{e}} \tau_{\mathrm{e}}\left(U_{\mathrm{G}_{0}}-c_{-}\right) \leqslant c_{0} \leqslant c_{+}+k^{2} v_{\mathrm{e}} \tau_{\mathrm{e}}\left(c_{+}-U_{\mathrm{G}_{0}}\right) .
$$

Since the flow is to be stable for disturbances of any wavelength, i.e. for all values of $k$, criterion [52] is obtained again. The wave velocities and the mean bubble velocity are sketched in figure 4 for $U_{L_{0}}=0$. Condition [59] is satisfied for $\epsilon_{0} \leqslant 0.39$. The instability occurs when the kinematic wave velocity becomes less than the lower of the two (modified) dynamic wave velocities. The critical value of the void fraction $\left(\epsilon_{0 \mathrm{cr}}\right)$ appears to be $35.3 \%$. From [60] it follows that the neutral stability curve is determined by

$$
c_{-}-k^{2} v_{\mathrm{e}} \tau_{\mathrm{e}}\left(U_{\mathrm{G}_{0}}-c_{-}\right)=c_{0} .
$$

The disturbances relevant to the instability are those of large wavelength.

The above analysis corroborates the idea put forward by Wallis (1969, Chap. 6) that the Lighthill-Whitham theory on instabilities arising out of a wave-hierarchy violation should be applicable to incompressible two-phase flows. The instability can be explained in the manner of Liu (1982) as arising out of "focusing" of void fraction perturbations along the lower-order waves owing to a negative diffusion coefficient, however it seems useful to also have an interpretation of 


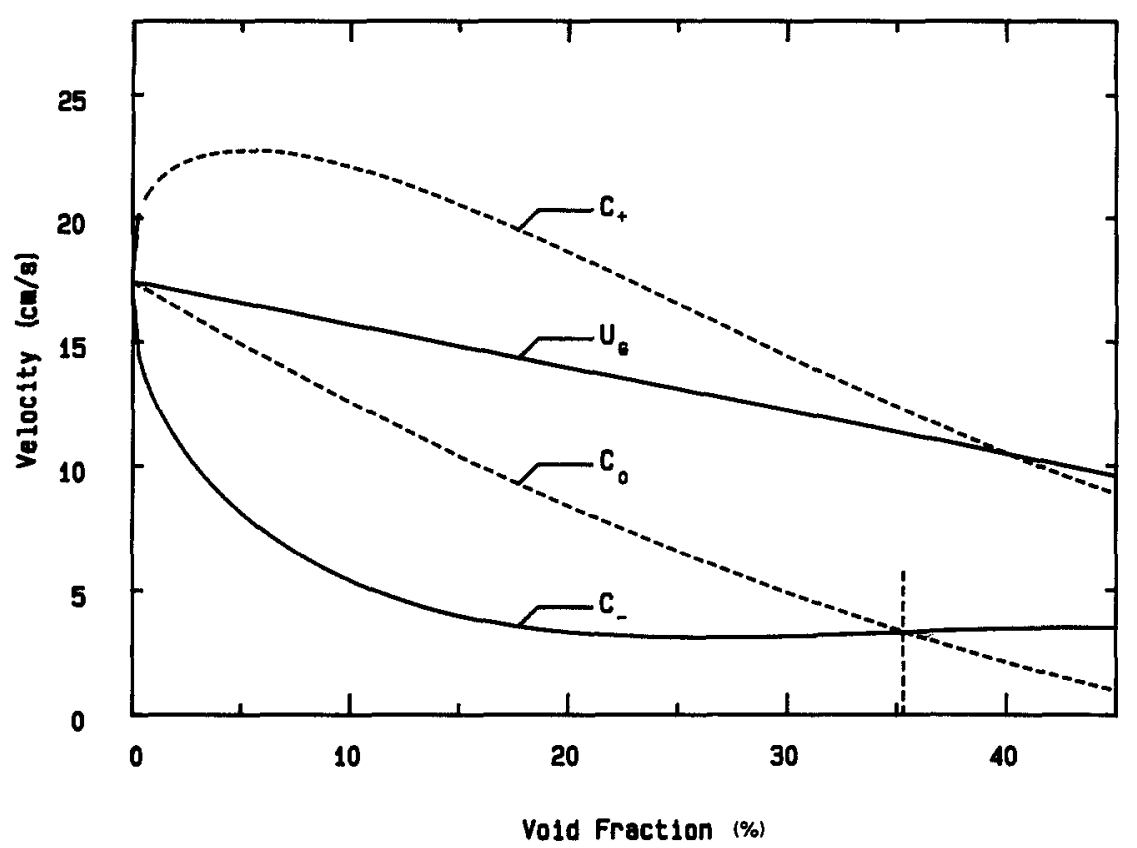

Figure 4. The mean bubble velocity $\left(U \mathrm{G}_{0}\right)$, the kinematic wave velocity $\left(c_{0}\right)$ and the dynamic wave velocities $\left(c_{-}\right.$and $\left.c_{+}\right)$, in stagnant fluid. The uniform flow is unstable when $c_{0}<c_{-}$, i.e. for $\epsilon_{0}>35.3 \%$.

the instability in terms of the dynamics of the bubble motion. Evaluation of [52] in terms of the flow parameters shows that the condition for stability is given by

$$
\left(\epsilon_{0} v_{0}^{\prime}+\frac{\frac{1}{4} \epsilon_{0} \rho_{\mathrm{L}} m_{0}^{\prime} v_{0}}{\rho_{\mathrm{G}}+\frac{1}{2} \rho_{\mathrm{L}} m_{0}}\right)^{2} \leqslant \frac{p_{\mathrm{e}}^{\prime}}{\rho_{\mathrm{G}}+\frac{1}{2} \rho_{\mathrm{L}} m_{0}}+\frac{\delta_{\mathrm{e}}}{\tau_{\mathrm{e}}}+\left(\frac{\frac{1}{4} \epsilon_{0} \rho_{\mathrm{L}} m_{0}^{\prime} v_{0}}{\rho_{\mathrm{G}}+\frac{1}{2} \rho_{\mathrm{L}} m_{0}}\right)^{2},
$$

which is identical to Batchelor's criterion for the (in)stability of uniform fluidized beds (expression [4.12] in his paper,) but for a differently defined effective pressure $p_{\mathrm{e}}$. An alternative form which more clearly indicates why the flow loses its stability is

$$
-\epsilon_{0}^{2} v_{0}^{\prime}\left[\left(\rho_{\mathrm{G}}+\frac{1}{2} \rho_{\mathrm{L}} m_{0}\right) v_{0}\right]^{\prime} \geqslant p_{\mathrm{e}}^{\prime}-\frac{12 \pi a \mu_{\mathrm{L}}}{\frac{4}{3} \pi a^{3}} f_{0} \delta_{\mathrm{e}} .
$$

The contributions to [61] are presented in table 1; for $\epsilon_{0}$ near $\epsilon_{0 \mathrm{cr}}$ each of the terms appear to be of the same order. The instability may be interpreted as follows. Consider a small perturbation of very long wavelength to a uniform flow. The local mean velocity of the bubbles is then $v \simeq U_{0}+v_{0}(\epsilon)$, and in the kinematic wave approximation the perturbation will propagate without attenuation or amplification relative to the bubbles with a velocity $\epsilon v_{0}^{\prime}(\epsilon) \simeq \epsilon_{0} v_{0}^{\prime}\left(\epsilon_{0}\right)$. Next, consider how the inertia of the bubbles and the surrounding fluid as well as the fluctuating bubble motion, due to hydrodynamic interactions or to liquid turbulence, affects the wave. A slight displacement of an element of bubbles would require a force $-\epsilon_{0}^{2} v_{0}^{\prime}\left[\left(\rho_{\mathrm{G}}+1 / 2 \rho_{\mathrm{L}} m_{0}\right) v_{0}\right]^{\prime} \partial \epsilon / \partial z$ in order to change the Kelvin impulse of the bubbles. As follows from table $1,-\epsilon_{0}^{2} v_{0}^{\prime}\left[\left(\rho_{\mathrm{G}}+1 / 2 \rho_{\mathrm{L}} m_{0}\right) v_{0}\right]^{\prime}$ is negative for $\epsilon_{0} \geqslant 0.25$, meaning that bubbles will be attracted to regions of larger void fraction and consequently that the uniform flow is unstable, if no other stabilizing force is present that drives bubbles down a void fraction gradient. This force is supplied by the effective compressibility of the bubbly fluid, due to the random bubble motions, that gives rise to a force $-\left[p_{\mathrm{e}}^{\prime}+9 \pi \mu_{\mathrm{L}} f_{0}\left(\epsilon_{0}\right) \delta\left(\epsilon_{0}\right) / a^{2}\right] \partial \epsilon / \partial z$. The pressure term is negative for $\epsilon_{0} \geqslant 0.31$, i.e. effective bubble pressure is then destabilizing. For $\epsilon_{0} \geqslant 0.35$ the stabilizing force is insufficient to prevent elements of bubbles to become attracted to regions of larger void fraction, and the flow is unstable. Note that we have ignored the contribution of the dynamic pressure forces on the bubbles to the effective pressure $p_{\mathrm{e}}$. As argued in section 5 this contribution is negative and its absolute magnitude will grow with increasing value of the void fraction. These forces will therefore promote the instability and cause it to occur at the smaller value of $\epsilon_{0 \mathrm{cr}}$ than estimated above. 
Table 1. Contributions to the stability criterion $[61]$ in $10^{-3} \mathrm{~N} / \mathrm{m}^{2}$

\begin{tabular}{ccccc}
\hline$\epsilon_{0}$ & $-\epsilon_{0}^{2} v_{0}^{\prime}\left[\left(\rho_{\mathrm{G}}+\frac{1}{2} \rho_{\mathrm{L}} m_{0}\right) v_{0}\right]$ & $p_{\mathrm{e}}^{\prime}$ & $\frac{12 \pi a \mu_{\mathrm{L}}}{\frac{4}{3} \pi a^{3}} f_{0} \delta_{\mathrm{e}}$ & Sum \\
\hline 0.20 & 0.2 & 2.5 & 1.8 & 4.5 \\
0.21 & 0.2 & 2.3 & 1.9 & 4.4 \\
0.22 & 0.1 & 2.2 & 1.9 & 4.2 \\
0.23 & 0.1 & 2.0 & 1.9 & 3.9 \\
0.24 & 0.1 & 1.7 & 1.9 & 3.7 \\
0.25 & 0.0 & 1.5 & 1.9 & 3.4 \\
0.26 & -0.1 & 1.3 & 1.9 & 3.2 \\
0.27 & -0.1 & 1.0 & 1.9 & 2.9 \\
0.28 & -0.2 & 0.8 & 1.9 & 2.5 \\
0.29 & -0.3 & 0.6 & 2.0 & 2.2 \\
0.30 & -0.4 & 0.3 & 2.0 & 1.9 \\
0.31 & -0.5 & 0.1 & 2.0 & 1.5 \\
0.32 & -0.6 & -0.2 & 2.0 & 1.2 \\
0.33 & -0.7 & -0.4 & 2.0 & 0.8 \\
0.34 & -0.8 & -0.7 & 1.9 & 0.5 \\
0.35 & -1.0 & -0.9 & 1.9 & 0.1 \\
0.36 & -1.1 & -1.1 & 1.9 & -0.3 \\
\hline
\end{tabular}

Positive contributions cause damping of the waves, negative contributions are destabilizing. The uniform flow is unstable for $\epsilon_{0} \geqslant 0.353$, when the sum of the three contributions is negative.

Figure 5 shows the growth rate $\left(k c_{i}\right)$ as a function of the wavenumber for values of the void fraction close to the critical value, where we have used the positive root of [56]. For void fractions of 0.4 and $0.5 \%$ above the critical void fraction (35.3\%) the maximum growth occurs at wavenumbers 234 and $261 \mathrm{~m}^{-1}$, respectively. In stagnant liquid the corresponding phase velocities are 3.27 and $3.26 \mathrm{~cm} / \mathrm{s}$ and so the frequencies of the fastest growing modes are 1.22 and $1.35 \mathrm{~Hz}$, respectively. A gain factor of 5 is obtained after the disturbances have moved upwards over a length of $181 \mathrm{~cm}$ in the first case, and $117 \mathrm{~cm}$ in the second.

It is tempting to think that the frequency of the fastest growing mode is the dominant frequency that will be observed in the experiments on unstable bubbly flows. As was found by Matuszkiewicz et al. (1987) this frequency increases with increasing value of the void fraction. Also, the slow

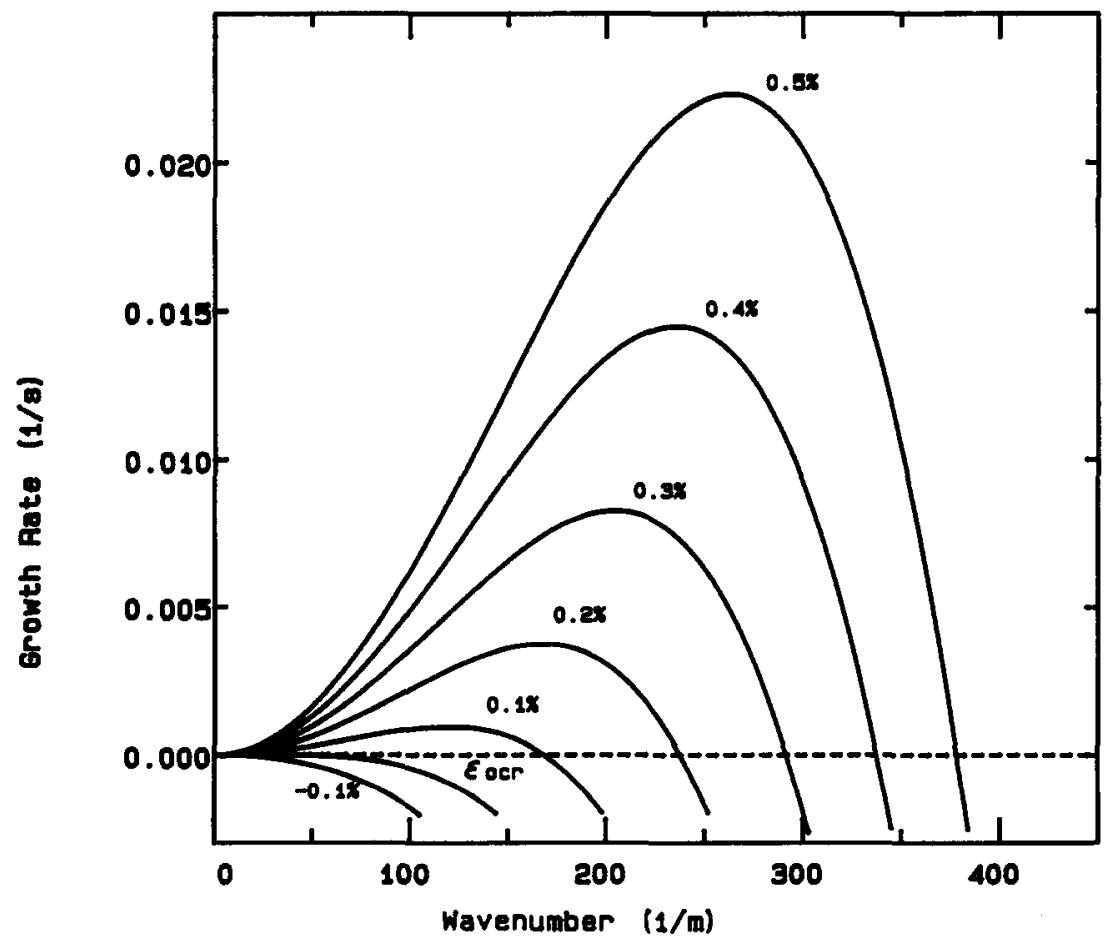

Figure 5. Growth rate of the unstable void fraction waves as a function of the wavenumber for various values of the void fraction above the critical value $(35.5 \%)$. The wavenumber of the fastest growing wave appears to increase with increasing value of the void fraction. 
growth of the disturbances conforms to their conclusion that the actual appearance of gas slugs entails a transition length, which will be shorter the larger the value of $\varepsilon_{0}-\epsilon_{0 \mathrm{cr}}$. It should be remarked that for a superficial liquid velocity of $0.18 \mathrm{~m} / \mathrm{s}$ the predicted frequency corresponding to the fastest growing mode is $13.0 \mathrm{~Hz}$ when $\varepsilon_{0}-\epsilon_{0 \mathrm{cr}}=0.5 \cdot 10^{-2}$, much higher than that observed by Matuszkiewicz et al. (1987).

\section{CONCLUDING REMARKS}

An approximate theory has been developed for the propagation of planar void fraction waves in uniform bubbly fluids. The equations of motion contain flow parameters that are determined by the statistics of the bubble motion. Since not much information regarding these statistics is available, simple models have been used for these parameters. Nonetheless, predictions of the theory are in qualitative agreement with the observations of void fraction waves by Mercadier (1981) and Micaelli (1982). The theory also shows that above some critical value of the void fraction a uniform bubbly fluid is linearly unstable to void fraction disturbances. Whether this instability is associated with the bubble-slug flow pattern transition, as the experiments of Matuszkiewicz et al. (1987) suggest, cannot be established by the present linear analysis.

Liu (1983) has shown by a weakly non-linear instability analysis of the one-dimensional equations that govern the propagation of voidage waves in fluidized beds, that a uniformly fluidized bed may develop quasi-steady periodic waves by a non-linear equilibration of the growing amplitude of linearly unstable disturbances. This can also be understood as a restoration of the stability due to a non-linear modification of the lower-order and higher-order wave velocities, such that a condition like [52] is again satisfied. Whether a similar equilibration process occurs in bubbly flows is presently under investigation.

The present analysis considers the stability of uniform bubbly flows to planar disturbances. Clearly, the transition to slug flow will be influenced by non-uniformities over the cross-section of the tube induced by the presence of the walls. There also is little doubt that the interference of planar disturbances with cross-stream disturbances is relevant to the transition process; there is need for further research here.

Acknowledgements - The authors would like to thank L. R. Seinstra for his assistance with the experiments. Aspects of this work were published in Biesheuvel (1984), and were presented at the ICHMT 1987 Int. Semin. on Transient Phenomena in Multiphase Flow, Dubrovnik, Yugoslavia (van Wijngaarden \& Biesheuvel 1987).

\section{REFERENCES}

Batchelor, G. K. 1967 An Introduction to Fluid Dynamics. Cambridge Univ. Press, Cambs.

BATCHELOR, G. K. 1988 A new theory of the instability of a uniform fluidized bed. J. Fluid Mech. 193, 75-110.

BIESHEUVEL, A. 1984 On void fraction waves in dilute mixtures of liquid and gas bubbles. Ph.D.

Thesis, Univ. of Twente, Enschede, The Netherlands.

Biesheuvel, A. \& Spoelstra, S. 1989 The added mass coefficient of dispersions of gas bubbles in liquid. Int. J. Multiphase Flow 15, 911-924.

Biesheuvel, A. \& VAN WiJngaARDEN, L. 1982 The motion of pairs of gas bubbles in a perfect fluid.

J. Engng Math. 16, 349-365.

GoldsteIN, H. 1980 Classical Mechanics, 2nd edn. Addison-Wesley, New York.

HetSRoni, G. (Ed.) 1982 Handbook of Multiphase Systems. Hemisphere, Washington, D.C.

Hirschfelder, J. O., Curtiss, C. F. \& Bird, R. B. 1954 Molecular Theory of Gases and Liquids. Wiley, New York.

KLuwick, A. 1977 Kinematische Wellen. Acta mech. 26, 15-46.

KLUwICK, A. 1983 Small-amplitude finite-rate waves in suspensions of particles in fluids. $Z$. angew. Math. Mech. 63, 161-171.

KYNCH, G. J. 1952 A theory of sedimentation. Trans. Faraday Soc. 48, 166-176.

Lighthill, M. J. 1986 An Informal Introduction to Theoretical Fluid Mechanics. OUP, Oxford. 
Lighthill, M. J. \& Whitham, G. B. 1955 On kinematic waves. I. Flood movement in long rivers. Proc. R. Soc. Lond. A229, 281-316.

LIU, J. T. C. 1982 Note on a wave-hierarchy interpretation of fluidized bed instabilities. Proc. $R$. Soc. Lond. A380, 229-239.

LiU, J. T. C. 1983 Nonlinear unstable wave disturbances in fluidized beds. Proc. R. Soc. Lond. A389, 331-347.

Matuszkiewicz, A., Flamand, J. C. \& Bouré, J. A. 1987 The bubble-slug flow pattern transition and instabilities of void fraction waves. Int. J. Multiphase Flow 13, 199-217.

MERCADIER, Y. 1981 Contribution à l'etude des propagations de perturbations de taux de vide dans les écoulements diphasiques eau-air à bulles. Thèse, Univ. Scientifique et Médicale et Inst. National Polytechnique de Grenoble, France.

MiCaelli, J.-C. 1982 Propagation d'ondes dans les écoulements diphasiques à bulles à deux constituants. Étude théorique et expérimentale. Thèse, Univ. Scientifique et Médicale et Inst. National Polytechnique de Grenoble, France.

MOORE, D. W. 1963 The boundary layer on a spherical gas bubble. J. Fluid Mech. 16, 161-176.

Needham, D. J. \& Merkin, J. H. 1984 A note on the stability and the bifurcation to periodic solutions for wave-hierarchy problems with dissipation. Acta mech. 54, 75-85.

NoORDZIJ, L. \& VAN WIJNGAARDEN, L. 1974 Relaxation effects, caused by relative motion, on shock waves in gas-bubble/liquids mixtures. J. Fluid Mech. 66, 115-143.

Rice, S. A. \& Gray, P. 1965 The Statistical Mechanics of Simple Liquids. Wiley, New York.

Wallis, G. B. 1969 One-dimensional Two-phase Flow. McGraw-Hill, New York.

WhithaM, G. B. 1959 Some comments on wave propagation and shock wave structure with application to magnetohydrodynamics. Communs pure appl. Math. 12, 113-158.

Whitham, G. B. 1974 Linear and Nonlinear Waves. Wiley, New York.

VAN WiJngaARDEN, L. \& Biesheuvel, A. 1987 Voidage waves in mixtures of liquid and gas bubbles. In Transient Phenomena in Multiphase Flow (Edited by AfGAN, N.). Hemisphere, Washington, D.C.

Zuber, N. 1964 On the dispersed two-phase flow in the laminar flow regime. Chem. Engng Sci. 19, 897-917. 